\title{
Investigación geoarqueológica en los depósitos de ocupación musterienses del Pleistoceno superior del Abric Romaní (Capellades, Barcelona, España)
}

\author{
J. Vallverdú i Poch ${ }^{(1,2 \text { y } 3)}$ \\ (1) IPHES, Institut Català de Paleoecologia Humana i Evolució Social, C/ Marcel.lí Domingo s/n- Campus Sescelades URV \\ (Edifici W3), E-43700 Tarragona, España \\ (2) Área de Prehistoria, Universitat Rovira i Virgili, Avda. Catalunya 35, E-43002-Tarragona, España \\ (3) Unidad asociada al CSIC, Departamento de Paleobiología, Museo Nacional de Ciencias Naturales. Calle José Gutierrez \\ Abascal 2, E-28006 Madrid, España \\ jvallverdu@iphes.cat
}

\begin{abstract}
RESUMEN
Los cambios en los objetivos de investigación y avances en los conocimientos científicos han diversificado la colaboración entre las ciencias de la Tierra, la Arqueología y la Historia. La Geoarqueología actualmente desarrolla un núcleo de investigación centrado en los sedimentos antrópicos de los yacimientos y de los paisajes arqueológicos. El potencial de los resultados sobre la investigación de los sedimentos antrópicos fue formulado en el último tercio del siglo XX. En la actualidad el estudio de los sedimentos antrópicos es una vigorosa línea de investigación basada en análisis empíricos multiescalares, como el que intenta ofrecerse en este estudio. En este trabajo presento una descripción sedimentológica de los depósitos de ocupación a través de los sedimentos formados durante el uso del fuego en el nivel $\mathrm{N}$ del Abric Romaní. Los datos son descripciones sedimentarias de las estructuras de combustión usando la documentación de campo y muestras en láminas delgadas. El objetivo de esta investigación es argumentar la realidad física de los depósitos de ocupación en el registro sedimentario de los yacimientos y los paisajes arqueológicos. La principal conclusión que propongo es la importancia del conocimiento sobre los asentamientos históricos y prehistóricos para guiar el registro de campo y el muestreo de estos archivos sedimentarios únicos. La finalidad de la investigación en el Abric Romaní es documentar la autoecología y la paleoecología de los grupos humanos arcaicos mediante el registro arqueológico.
\end{abstract}

Palabras clave: fuego, lámina delgada, microestratigrafia, Musteriense, Pleistoceno superior.

\section{The geoarchaelogical research on the Late Pleistocene Mousterian occupation deposits of the Abric Romaní rock shelter (Capellades Barcelona, Spain)}

\begin{abstract}
Changes in the focus of such research in conjunction with scientific advances have diversified the relationship between Earth sciences, Archaeology and History. Today, the core concept of geoarchaeology focuses on the study of anthropic sediments recovered from archaeological sites and landscapes. The potential results that anthropic sediments can offer were highlighted in the last third of the 20th century. Currently, the study of anthropic sediments is a dynamic field within archaeological research focusing on multi-scale analyses such as I try to present in this paper. Here, I give a sedimentary description of the living floors using sediments associated with the use of fire in the archaeological bed north of the Abric Romani rock-shelter site. The data are the lithological descriptions of the combustion structures taken from the field record and thin section samples. The objective is to provide observations to demonstrate the physical reality of the living floors in the sedimentary record of archaeological sites and landscapes. My main conclusion highlights the importance of knowledge about historic and prehistoric human settlements in order to guide the field record and sampling of these rare sedimentary records. The aim of the research on the Romani rock shelter is to report on the paleoecology and the autoecology of the archaic human groups through the archaeological record.
\end{abstract}

Key words: fir, Late Pleistocene, microstratigraphy, Mousterian, thin section. 
Vallverdú i Poch, J., 2018. Investigación geoarqueológica en los depósitos de ocupación... Boletín Geológico y Minero, 129 (1/2): $129-152$

\section{ABRIDGED ENGLISH VERSION}

\section{Introduction}

The sedimentary succession of the Abric Romaní rock shelter (Fig. 1) contains a Late Pleistocene archaeological bed sets. U-series dating confines the chronology of the archaeological beds to between 60 and $40 \mathrm{ky}$ $B P$, in the early and middle MIS 3 (marine isotopic stage 3) (Fig. 2) (Bischoff et al., 1988; Vaquero et al., 2013). The archaeological beds are stratified between scree deposits and biochemical deposits (speleothems and travertines) near the wall of the rock shelter. These chemical and rock fall deposits, with the contribution of water flow deposits, constitute a $40 \mathrm{~m}$ thick talus cone at the foot of a travertine cliff. The archaeological beds are thin and contain combustion structures, wood imprints, faunal and charcoal remains, and Mousterian lithic implements. The prehistoric settlements at Abric Romani shelter are related to the last archaic human groups (the Neanderthal lineage) and the first arrivals of modern human groups on the Iberian Peninsula.

In this contribution, the geoarchaeological inquiry considers the study of the cultural deposits and especially of the occupation deposits preserved in the stratigraphic contexts of archaeological sites. The objective is provide evidence of the physical reality of anthropic sediments and their study adds to empirical archaeological research focusing on recognizing the existence of living floors in the archaeological record. Archaeologists are interested in living floors as a means of explaining behavioural inferences based on the evidence provided by the spatial distribution of archaeological remains and structures (Bordes, 1975; Dibble et al., 1997; Schiffer, 1987b). This archaeological approach consists of determining the site structure patterns of human settlements based on the archaeological record. The first inquiries come from prehistoric archaeology for comparing distinctive behavioural signatures applicable to theories regarding the evolution of the genus Homo.

Advances in interdisciplinary work on archaeological projects (archaeometry, bioarchaeology, and geoarchaeology) have promoted results based on anthropic sediment analyses.

\section{Materials and methods}

This study attempts to describe a part of the site structure of archaeological bed $\mathrm{N}$ using the anthropic sediments related to the use of fire (Vallverdú i Poch et al., 2010). The archaeological assemblage of bed $N$ contains combustion structures, one wood imprint, faunal and charcoal remains, and Mousterian knapped stones (mainly made from chert). Five combustion structures, empty of faunal and lithic remains, were located near the northwestern wall of the rock shelter. The five combustion structures are arranged at a distance of one meter from one another (Figs. 3 and 4). The spatial distribution of these five combustion structures suggest an analogy with the sleeping areas reported in prehistoric ethnology and in the ethnoarchaeological studies of the settlements of modern human groups (Vallverdú $i$ Poch et al., 2010). The sleeping areas, as well as the so-called 'resting or snack areas' (Binford, 1988), are a specific activity area of the residential settlement pattern (Binford, 1987).

The combustion structure sediments are described with the help of field (facies) and microscopic (microfacies) observations in accordance with terminology from sedimentary petrography and soil micromorphology (Figs. 5, 6 and 7). The anthropic sediments observed in the combustion structures are characterized in order to explain the thermal modifications and the formation of charcoal-rich beds attributed to the use of fire during the prehistoric settlement of the rock shelter. Another objective of this study is to elucidate the preservation and post-depositional transformations of the anthropic sediments based on microscopic observations (Fig. 7).

\section{Results}

Archaeological bed $\mathrm{N}$ includes two sedimentary microfacies: grey gravel and sand and brown-grey gravel and sand. The anthropic microfacies of the combustion structures contain dominant travertine rock fragments burned at different temperatures and by means of different combustion modes. The field description of the anthropic sediments of the combustion structures note, firstly the lineal dimension measured in the geometries of the plant-charcoal-rich beds, and secondly, the content (\%) of plant charcoal and burned travertine, and the thickness of the reddened and charcoal-rich beds. The correspondence between the microfacies and the field sedimentary facies descriptions seeks to determine the homogeneity or the heterogeneity of the heating phenomena in the travertine rock fragments.

The heterogeneity of the thermal modification of the travertine rock fragments observed in the anthropic microfacies provides evidence that the sedimentary beds sampled in the combustion structures were the pro- 
duct of different episodes of the human use of fire. The microscopic ashes are difficult to separate from the disintegrated microsparites of the fragments of calcined travertine. The microarchaeological study of the fuels used has identified wood charcoal, fragments of charred leaves with typical druse spherulites, and siliciclastic aggregates rich in iron oxides related to wood and bark (Weiner, 2010). The evidence of microstratified corrosion between masses of pseudomorphic calcite, crumbled from calcined travertine and ashes, points to episodes of fire use separated by weathering episodes. These sediments of the combustion structures are modified by surficial cryoturbation, calcification caused by dripping water, and human trampling (Figs. 6 and 7).

The sedimentary facies study based on the field observations describes differences in the thickness of the charcoal-rich beds. The plant-charcoal-rich beds of the combustion structures in the sleeping area are thinner than the other combustion structures in archaeological level $N$. Thin section examination partially confirms this difference. These differences points to distinct uses of fire and constructive techniques as a possible explanation for the different types and morphologies of combustion structures in the archaeological assemblage (Vallverdú i Poch et al., 2012).

\section{Discussion and conclusion}

The description of the anthropic sediments using sedimentary facies in fieldwork and sedimentary microfacies in thin sections attempts to maintain the continuity of the scale of observation. Thus, it is possible to suggest a certain degree of continuity in some field and microscopic sedimentary features: cut and fill sedimentary structures related to dripping water on combustion structures, and reddened sediments observed as opaque sediments in the thin sections. Meanwhile, for other sedimentary features and components it was impossible to find a link between the microscopic and field observations, such as the black charcoal-rich beds described in the fieldwork (Fig. 5).

Archaeological studies, and especially microarchaeological research, are specifically concerned with the characterization of the use of fire through combustion features in order to explain its first appearance and its implications for theories regarding the evolution of Homo (Mentzer, 2014). The differences of scale between the field observations of the combustion structures and the microscopic combustion features are inescapable and, in my opinion, call for a critical position in suggesting the differences between use of fire vs combustion structure in the archaeological record.

The preservation of evidence of fire use in the form of combustion structures in the sedimentary record is poorly understood. The preservation of the sedimentary signatures of the use of fire in archaeological sites is probably enhanced when anthropic burial is active, and may be related to the reuse of combustion structures, as in the Mousterian site of Kebara (Israel) or in the stabling floors of cave-enclosures. The archaeological research on the use of fire should begin by integrating the combustion structures in the continuity of the living floors of the archaeological bed. This continuity between the research of particular archaeological features (activity areas and constructions) and the structure of the human settlements of the past requires archaeological assemblages recorded over large surfaces.

Archaeological research focused on determining the use of fire provides solid evidence for exploring the site structure as well. This approach involves exploring the archaeological beds beyond their stratigraphy combined with geoarchaeology to support the description of specific archaeological features of the living floors, such as constructions and activity areas, using the foundations of geological cartography. Maps of anthropic sediments related to archaeological beds are rare in archaeological research at sites or ethnoarchaeological settlements (Fig. 8). Current archaeological research includes a large number of studies on cultural deposits with anthropic sediment types that are based on samples and observations of prehistoric, historic and experimental sites. Most of these anthropic sediments are described as sedimentary structures, e.g. the sedimentary crusts are related to the human compaction sampled in the archaeological beds (Fig. 7), as well as other features or components of the anthropic sediments, which provide sedimentary evidence that make it possible to specify the empirical reality of the living floors in the archaeological record. The microstratigraphy of anthropic sediments have helped us to differentiate, as in the combustion structures of the Abric Romani archaeological bed N (Vallverdú i Poch et al., 2010), the dimensional attributes of charcoal-rich lenticular beds noted in the field (by the naked eye observations) using sedimentary facies analyses (Fig. 8).

\section{Introducción}

El estudio de los sedimentos del Abric Romaní demuestra la posibilidad de abordar problemas centrales de la investigación arqueológica con la ayuda de las ciencias de la Tierra. Se trata de la caracterización de las actividades y las conductas de las poblaciones humanas mediante el registro arqueológico. El estudio de los sedimentos que abordamos en este trabajo sostiene la existencia de una continuidad y 
unidad conceptual entre las investigaciones dedicadas a la Arqueología de las poblaciones arcaicas (anteriores a las poblaciones humanas modernas) y a la Arqueología de las poblaciones modernas (prehistóricas e históricas). Esta continuidad y unidad ha tenido sin embargo bifurcaciones conceptuales $y$ metodológicas específicas para plantear y resolver diferentes líneas de investigación en Arqueología (Renfrew, 1976): la Arqueometría focalizada en las ciencias físicas y químicas para determinar, por ejemplo, la datación, procedencia o composición de los restos arqueológicos; la Bioarqueología, bajo el auspicio de las ciencias biológicas y no sólo como zoólogos y botanistas, sino con dos disciplinas denominadas Arqueozoología y Arqueobotánica. La contribución de las ciencias de laTierra en las investigaciones arqueológicas han sido agrupadas en el término de Geoarqueología y su objeto de trabajo trata los sedimentos y paisajes (formas) que contienen los restos arqueológicos.

La estratigrafía de los yacimientos arqueológicos es una investigación que a menudo es llevada a cabo por geólogos en muchos proyectos de investigación. La llegada de las dataciones numéricas ha fundamentado la aparición de la Geocronología, afín a las disciplinas agrupadas en torno a la Arqueometría, pero aún continúa siendo el objetivo primario de muchos geoarqueólogos el establecer la estratigrafía de los yacimientos: la ordenación temporal de su sucesión sedimentaria. El hecho de que muchos proyectos de investigación paleoantropológicos dispongan de un cuadro cronoestratigráfico solvente ha atraído a muchos geocientíficos interesados en depósitos sedimentarios recientes. Los depósitos del Cuaternario han dejado de ser sedimentos que dificultan la observación de los afloramientos geológicos (Solé Sabarís, 1956). El estudio de sucesiones sedimentarias y formas del paisaje, formadas dentro de la escala temporal del Cuaternario, son una guía para la analogía "actualista" en Geología sedimentaria.

Una segunda bifurcación prominente en el desarrollo de la práctica y de la teoría geoarqueológica se basa en la investigación de los sedimentos, suelos y paisajes arqueológicos para caracterizar las actividades y conductas de las poblaciones humanas (Butzer, 1989; Goldberg and Mcphail, 2006; Mattews, 1994; Shahack-Gross, 2008; Stein, 2001; Stein y Farrand, 1985; Weiner, 2010). Esta línea de investigación basada en la formación y modificación antrópica de los depósitos sedimentarios, tanto detríticos como químicos, marca una prioridad en los objetivos actuales de las investigaciones geoarqueológicas hacia la autoecología y la paleoecología de las poblaciones humanas. Entre los procesos sedimentarios de for- mación de yacimientos arqueológicos se distinguen los procesos naturales y los procesos culturales (Schiffer, 1987b). Desde un punto de vista tautológico, la identificación de los procesos culturales en los depósitos arqueológicos precisa también el descarte de los procesos sedimentarios naturales.

Este trabajo va a ser dedicado a presentar los estudios geoarqueológicos del Abric Romaní y trata de exponer los principales resultados sobre los procesos sedimentarios culturales determinados en sus depósitos de ocupación musterienses. El interés de los arqueólogos por la investigación del comportamiento de los grupos humanos fue iniciado de manera fundamental por la Arqueología prehistórica. Hay numerosos antecedentes, pero su formulación teórica y práctica corre paralela a la renovación teórica y metodológica llevada a cabo principalmente por la Etnología prehistórica francesa y la Nueva Arqueología americana a finales de los años 60. Durante estos 50 años que nos separan de esta renovación en la investigación arqueológica, ha sido privilegiada la documentación de los llamados depósitos de ocupación y el análisis espacial de la distribución del registro arqueológico. Esta nueva orientación de la Arqueología ha alimentado muchas investigaciones. Hay arqueólogos y equipos de investigación optimistas o pesimistas que tratan de la posibilidad de documentar registros arqueológicos con y sin valor para el análisis de la conducta de las poblaciones humanas (Schiffer, 1987b). La verificación de las estructuras y conjuntos de restos arqueológicos, en posición primaria y en superficies de excavación de gran tamaño, ha permitido construir esta línea de investigación activa y foco de debate en Arqueología. $Y$ especialmente se ha expandido hacia la Arqueología de periodos históricos y la Arqueología experimental (March et al., 2014). La Arqueología de las poblaciones humanas arcaicas llevada a cabo en el Abric Romaní es un proyecto de investigación reconocido internacionalmente (Balter, 2009).

\section{Área de estudio}

El yacimiento del Abric Romaní es un gran abrigo situado en la vertiente Norte del acantilado travertínico de la Cinglera del Capelló (Capellades, comarca de la Anoia, provincia de Barcelona) a 300 m s.n.m. El acantilado de la Cinglera es un escarpe rocoso de 60 $\mathrm{m}$ de espesor formado en el flanco oriental de una mesa de travertino sobre la que se asienta la localidad de Capellades (Fig. 1). Esta mesa travertínica se localiza en la margen derecha del rio Anoia, que en la zona del Abric Romaní discurre por un estrecho paso 
que une la depresión prelitoral del Penedès y la subcuenca de Òdena, una depresión marginal de la cuenca del Ebro. Delante del abrigo, el Anoia está encajado a 240 m s.n.m.

El acantilado de la Cinglera del Capelló de Capellades fue intensamente explorado por Amador Romaní, asociado con el Institut d'Estudis Catalans, durante el primer tercio del siglo XX (Bartroli et al.,
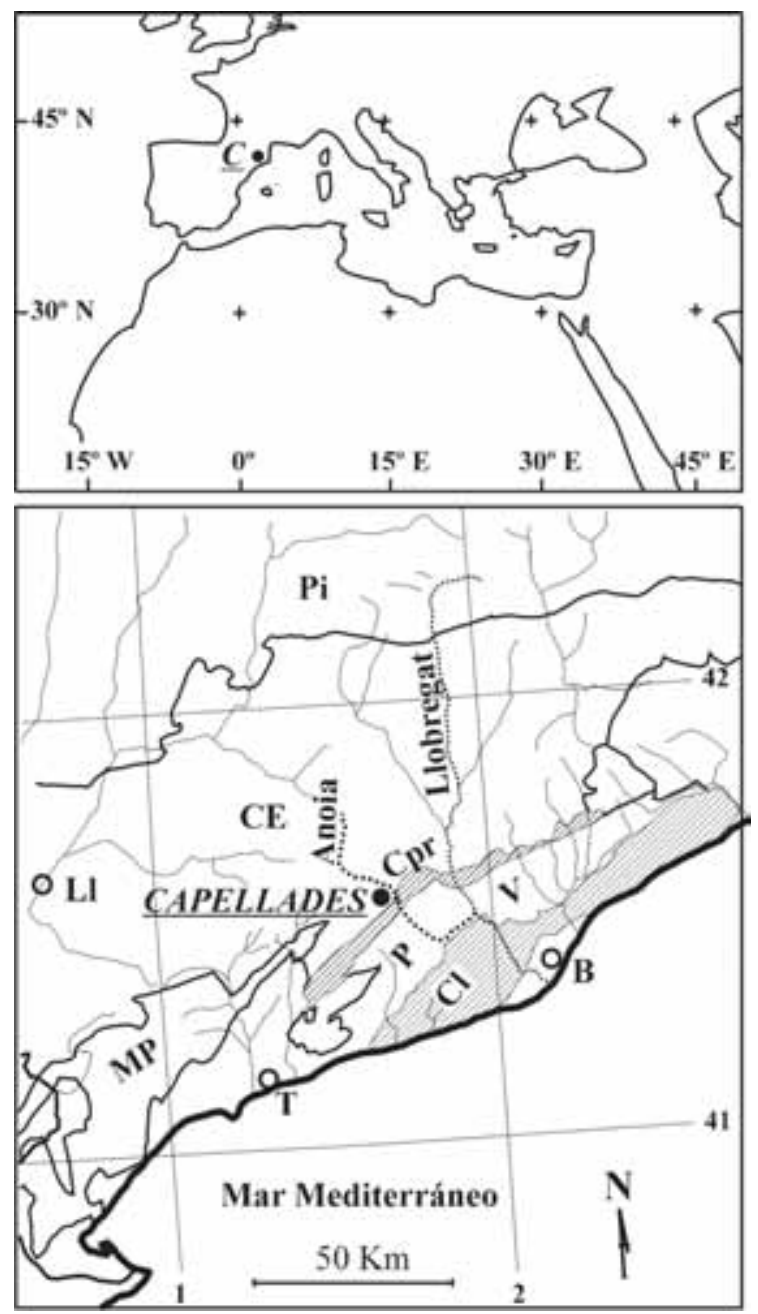

Figura 1. Localización geográfica de Capellades (provincia de Barcelona, España) y grandes unidades de la fisiografía del NE de la Península Ibérica.

Abreviaturas: Pi, Pirineos; $\mathrm{CE}$, cuenca del Ebro; $\mathrm{Cpr}$, cordillera prelitoral; $\mathrm{P}$, depresión del Penedès; $\mathrm{V}$, depresión del Vallès; $\mathrm{Cl}$, cordillera litoral; MP, macizo de Prades; LI, Lleida; $\mathrm{B}$, Barcelona; T, Tarragona.

Figure 1. The geographic position of Capelllades (province of Barcelona, Spain) and major physiographical units in the NE Iberian Peninsula.

Legend: Pi, Pyrenees; $C E$, Ebro Basin; Cpr, Pre-coastal ranges; $P$, Penedès graben; $V$, Vallès graben; $\mathrm{Cl}$, Coastal range; $\mathrm{MP}$, Prades massif; LI, Lleida; $B$, Barcelona; T, Tarragona.
1995; Vidal, 1913). El último período de excavaciones arqueológicas se inicia el 1983 hasta la actualidad. Las numerosas dataciones de los travertinos, mediante las series del uranio, y la conservación del polen han proporcionado datos muy relevantes para la estratigrafía y la paleoecología de las ocupaciones humanas del abrigo y de la región de Capellades (Bischoff et al., 1988; Burjachs y Julià, 1994). Hasta la actualidad se ha excavado una profundidad máxima de $11 \mathrm{~m}$ (Fig. 2). La superficie de excavación tiene una extensión abierta que oscila entre los $350 \mathrm{~m}^{2}$ y los 250 $\mathrm{m}^{2}$. El abrigo tiene un sondeo realizado por Amador Romaní que llegaba hasta los $12 \mathrm{~m}$ por debajo de los últimos travertinos de la sucesión estratigráfica (el Pozo 1). Este pozo ha sido profundizado $6 \mathrm{~m}$ más por el equipo de investigación actual. También existe un sondeo mecánico con sedimento recuperado en el que se ha evidenciado otros $30 \mathrm{~m}$ de depósitos por debajo de los $11 \mathrm{~m}$ excavados en extensión. Por lo tanto, el grosor estimado de la estratificación del talud a pie del acantilado es de unos $40 \mathrm{~m}$. La base del sondeo mecánico tiene una altitud cercana a los 260 $\mathrm{m}$ s.n.m. Esta cota es próxima a la elevación de la terraza $+20 /+25 \mathrm{~m}$ del río Anoia.

La litoestratigrafía del Abric tiene una zonación evidente provocada por la configuración de las cortinas calcíticas y de los alvéolos (tafonis) en la pared del acantilado. Las cortinas calcíticas o viseras, denominadas Capelló en la región de Capellades, se forman por la fijación de carbonato cálcico sobre la vegetación de estos escarpes calizos poblados por la comunidad botánica Adiantetea (Folch, 1981). Estas cortinas de la Cinglera del Capelló desarrollan morfologías sobre la pared del acantilado descritas como "arbol de navidad" (Salomon, 1997). A pie del acantilando travertínico, la pared es enterrada por los siguientes depósitos interestratificados:

1. Depósitos de caídas de rocas, compuestos por grandes bloques y gravas de travertino de cornisa y pared con arenas y limos calcíticos. Estos fragmentos de roca tienen relación con la meteorización de la pared y visera del abrigo por la fragmentación mecánica y la disgregación granular.

2. Depósitos de corrientes de agua, formadas por fragmentos de travertino de tamaño grava, arena y limo.

3. Depósitos de precipitación química: espeleotemas (sinter crusts) y travertinos de plantas impregnadas (plantas inferiores y superiores, algas, musgos, hepaticas, etc. en posición de vida o en fragmentos) (Julià, 1983).

4. Depósitos eólicos de composición calcítica y siliciclástica. 


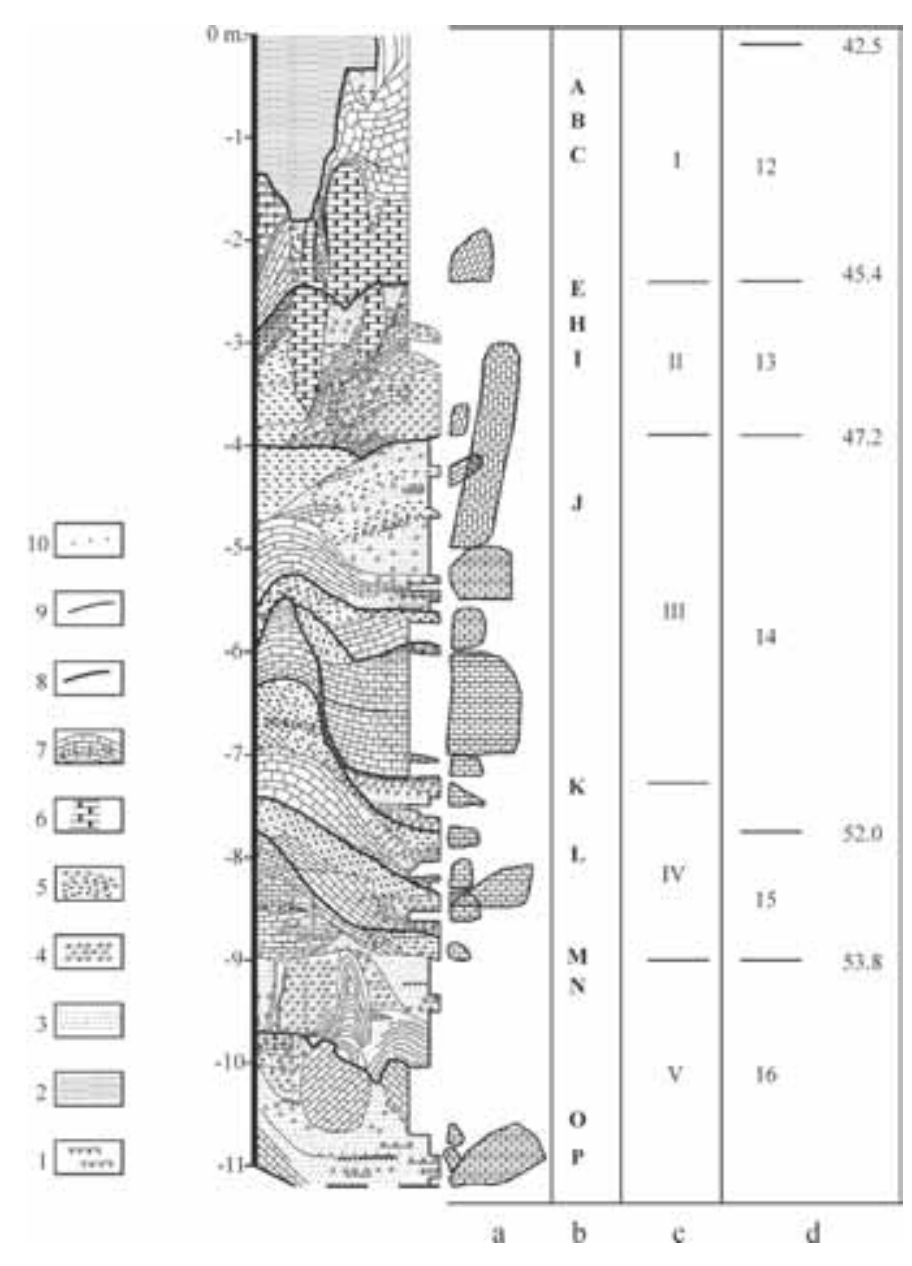

La sucesión sedimentaria descrita en el perfil de la Coveta Nord tiene $11 \mathrm{~m}$ de espesor y permite observar la evolución de la estratificación del talud del acantilado. El perfil puede ser dividido en dos grandes conjuntos sedimentarios: el inferior, caracterizado por la sedimentación clástica y química a pie de escarpe; y el superior, caracterizado por la sedimentación química y eólica dentro de una cavidad.

La estratificación del talud a en el Abric contiene dos litotopos mayores, adyacentes entre la pared y la línea de goteo del acantilado. La sucesión sedimentaria muestra una estratificación rítmica que sugiere el crecimiento y el retroceso de la cornisa del abrigo (Fig. 2). La acreción progradante de la cortina calcítica ha permitido estimar una gran superficie protegida debajo de la cornisa de hasta $15 \mathrm{~m}$ lineales entre la pared y la línea de goteo del abrigo. La acreción de la visera se produce en general cuando a pie de pared y en la línea de goteo son dominantes los depósitos de corrientes de agua y precipitación química. Por lo
Figura 2. Litoestratigrafía de la sección de la Coveta Nord del Abric Romaní. La columna estratigráfica contiene también la posición de los niveles arqueológicos en relación con las fechas de los límites basales de los acontecimientos Dansgaard-Oeschger según el modelo temporal del sondeo GISP 2 (Blunier and Brook, 2001). Un sumario actualizado de las fechas obtenidas por las series del uranio de los niveles arqueológicos del Abric Romaní ha sido publicado recientemente (Vaquero et al., 2013).

Leyenda de la columna litológica: 1, horizonte organomineral gris; 2 , arena limosa calcítica y siliclástica roja; 3 , arena calcítica amariIla; 4, gravas de travertino tobaceo con arenas calcíticas amarillas; 5 , gravas planas de travertino cristalítico con arenas y limos calcíticos de color amarillo blanquecino; 6, espeleotemas; 7, arenas cementadas y travertinos; 8, diastema; 9, paraconformidad o discordancia erosiva; 10 , lecho arqueológico.

Leyenda de las columnas de comentarios: a, caída de rocas de bloques y megabloques de travertino; $b$, letras de los niveles arqueológicos; d, secuencias sedimentarias; e, acontecimientos Dansgaard-Oeschger y las fechas de sus límites basales de acuerdo con el modelo de temporal del sondeo GISP2 (Blunier and Brook, 2001).

Figure 2. The lithostratigraphic column of the Abric Romani Coveta Nord profile. The stratigraphic column contains the position of the archaeological levels in accordance with the dates of the basal boundaries of the Dansgaard-Oeschger events in the GISP2 temporal scale model (Blunier y Brook, 2001). An updated summary of the $U$-series dates of the Abric Romani archaeological beds has recently been published (Vaquero et al., 2013).

Legend for the lithological column: 1, organomineral grey horizon; 2, red siliciclastic and calcitic silty sand; 3, yellow calcitic sand; 4, yellow tuffaceous-travertine gravel and calcitic sand; 5 , platy gravels of crystallitic travertine and calcitic sand and silt; 6, speleothems; 7, cemented sands and travertines; 8, diastem; 9, paraconformity or erosive unconformity; 10, archaeological bed. Legend for the comment columns: a, rock-fall of travertine blocks and megablocks; $b$, letters of the archaeological beds; e, sedimentary sequences; $d$, the dates of the lower boundaries of the Dansgaard-Oeschger events in the temporal scale model of the GISP2 core (Blunier y Brook, 2001).

tanto, se puede considerar que el sobrepeso y descompresión son los causantes de la caída de bloques documentados en la sucesión estratigráfica del Abric. Los períodos de retroceso de la cornisa son dominados por depósitos de fragmentación (gravitacional) y de precipitación química.

El conjunto superior de la sucesión estratigráfica del Abric es una cavidad cárstica formada por la fusión de la cortina de la visera con los depósitos de pie de escarpe. El relleno de la cavidad era un depósito eólico más o menos coluvionaldo y excavado en el primer tercio del siglo XX. Se trata de un depósito descrito como similar a los loess calizos (o loess-like) pleistocenos tal como dejó escrito el mismo Amador Romaní y otros investigadores posteriores (Arteaga et al., 2001b; Bartroli et al., 1995; Bischoff et al., 1988).

La cronología de la sucesión sedimentaria ha sido profusamente estimada en el Abric Romaní y, en menor medida, en la Cinglera del Capelló (Vaquero et al., 2013). La cronoestratigrafia de la Coveta Nord 
(Fig, 2) está limitada por los horizontes de 40 ka y 55 ka BP En la figura 2 no detallamos las fechas de las series del uranio medidas en los travertinos del Abric estratificados con los niveles arqueológicos (Vaquero et al., 2013), pero sí que presentamos los intervalos cronoestratigráficos que las incluyen. Los intervalos cronostratigráficos se basan en la cronología de los límites basales de los eventos Dansgaard-Oeschger del Preistoceno superior (con el modelo de edad del sondeo GISP2) (Blunier y Brook, 2001). Descripciones más detalladas de las litoestratigrafías y la cronología del Abric Romaní son accesibles en diferentes publicaciones (Bischoff et al., 1988; Bischoff et al., 1994; Carbonel et al., 1994; Giralt y Julià, 1996; Mora et al., 1988; Muro et al., 1987; Vallverdú i Poch et al., 2012; Vallverdú i Poch et al., 2005b; Vaquero et al., 2013).

Los depósitos arqueológicos del Abric Romaní se presentan dentro de la sucesión sedimentaria como estratos de espesor centimétrico $(1-20 \mathrm{~cm})$ de gran continuidad lateral. Estos depóstios suelen estar separados por espesos estratos sin restos arqueológicos (Mora et al., 1988; Vallverdú i Poch et al., 2005b). La microestratificación de los depósitos arqueológicos y la abundancia de las estructuras de combustión condujeron a los investigadores del último proyecto de intervención arqueológica a promover una excavación de gran superficie (Mora et al., 1988). Tal como hemos mencionado más arriba, la intervención en extensión en el Abric Romaní perseguía explicar la distribución de los restos arqueológicos mediante una aproximación basada en la Etnología prehistórica y la etnoarqueología impulsada por la Nueva Arqueología en España (Carbonell et al., 1983). Este paradigma sigue siendo el objetivo del actual proyecto de investigación 30 años después de trabajos de campo. Los resultados han sido publicados en revistas nacionales, internacionales y libros monográficos (Carbonell, 1992; Carbonell, 2002; Carbonell et al., 1996; Carbonell i Roura, 2012; Chacón et al., 2012).

\section{Métodos y teorías sobre el registro y la interpretación de los conjuntos arqueológicos del Abric Romaní}

La intervención arqueológica en extensión en el Abric Romaní se basa en los protocolos estandarizados de una excavación planimétrica (Julien, 2002). Se trata de registrar los restos y las estructuras arqueológicas mediante coordenadas tridimensionales; la microestratigrafía, documentación gráfica y topografía de estructuras arqueológicas (hogares, hoyos, muros, enlosados de roca, etc.); y la topografía del asentamiento prehistórico. Los depósitos arqueológicos del Abric Romaní son fáciles de distinguir de los depósi- tos sedimentarios naturales. Los depósitos de ocupación tienen un espesor variable y se disponen sobre una superficie relativamente continua. Por lo tanto, no se trata de depósitos arqueológicos en sedimentos homogéneos para los que hay también descritos protocolos de excavación (David et al., 1973). Los niveles arqueológicos del Abric Romaní tampoco son un único "suelo" de ocupación en el sentido descrito por Bordes: "una superficie reconocible sobre la cual ha vivido el hombre paleolítico... en la que se pueda esperar a deducir de la posición de los restos alguna cosa entorno a sus actividades." (Bordes, 1975). En la actualidad, los niveles arqueológicos del Abric Romaní son considerados palimpsestos y contienen un número indeterminado de depósitos culturales estratificados con depósitos naturales junto con restos culturales sin una relación necesaria con su grosor (Bailey, 2007; Dibble et al., 1997).

Las investigaciones publicadas que han tratado de alentar o prevenir la interpretación de la distribución espacial de los restos y estructuras arqueológicas son muy abundantes en la literatura arqueológica y etnoarqueológica. Las aproximaciones basadas en la distribución de las estructuras arqueológicas, en cierto modo, han sido más exitosas para caracterizar las áreas de actividad en los asentamientos prehistóricos. Este es el caso del registro de las estructuras de combustión del Abric Romaní tal como veremos en los resultados.

La interpretación de la distribución espacial de los restos arqueológicos de rocas talladas, fragmentos de huesos y otros restos (incluyendo microrrestos) es actualmente más controvertida en el estado de la cuestión de esta línea de investigación (Bailey, 2007; Dibble et al., 1997; Vaquero, 2013). Este paradigma sugiere que los restos arqueológicos sólo explican episodios de actividad más que áreas de actividad humanas en los depósitos de ocupación. La existencia de áreas de actividad humana es difícil de demostrar mediante los datos empíricos. A menudo estas investigaciones van acompañadas de la descripción de procesos de formación y modificaciones naturales, o episodios de reciclaje de restos líticos que distorsionan la distribución espacial y la contemporaneidad de las áreas de actividad en la distribución espacial. Las informaciones aportadas por los remontajes de las rocas talladas alertan de la necesidad de demostrar la contemporaneidad de los episodios de actividad (Vaquero, 2008; Vaquero, 2013; Vaquero et al., 2015). La distribución espacial de los restos de fauna y de los carbones ofrece mayor confianza para determinar áreas de actividad. La posibilidad de utilizar de nuevo estos tipos de restos y formar un área de actividad sobrepuesta se contempla como menos probable (Dibble et al., 1997; Schiffer, 1987b). 
Vallverdú i Poch, J., 2018. Investigación geoarqueológica en los depósitos de ocupación... Boletín Geológico y Minero, 129 (1/2): $129-152$

Los últimos paradigmas teóricos desarrollados en la investigación de los conjuntos arqueológicos del Abric se centran en la noción de palimpsesto y la existencia de episodios discretos de actividad (Chacón et al., 2015; Vaquero, 2008; Vaquero et al., 2012). El uso de remontajes de rocas talladas y quemadas, y de restos de fauna son la entidad empírica más sólida para interpretar la organización espacial de los restos. Esta aproximación sugiere la posibilidad de reconstruir, a partir acontecimientos individuales (Gowlett, 1997), parte de la causalidad en la distribución espacial del conjunto arqueológico documentado.

Las estructuras arqueológicas documentadas en el Abric son en su mayoría estructuras de combustión. En particular, las estructuras de combustión del Abric han sido tratadas mediante dos aproximaciones metodológicas distintas. La primera, el uso del fuego ha sido considerado por ser un área de actividad caracterizada por el consumo final de combustible (vegetal, etc.), de forma análoga a otros restos del conjunto arqueológico. Los residuos de la combustión se acumulan discretamente junto a otros restos (líticos y faunísticos) y otras estructuras (bloques de travertino, hoyos, cubetas, etc.) para formar un conjunto arqueológico relacionado con un hogar (hearth related assemblages) (Vallverdú i Poch et al., 2005a; Vaquero y Pasto, 2001). Esta acumulación formada por el uso del fuego es útil para ser considerada una entidad para el análisis espacial. Esta aproximación heurística de la organización espacial del uso del fuego y su relación con los restos arqueológicos se ha visto debilitada a medida que ha aumentado el número de niveles arqueológicos excavados. No ha sido posible utilizar este tipo de unidades espaciales en depósitos de ocupación donde los conjuntos arqueológicos forman una acumulación continua con muchas estructuras de combustión; o la evidencia empírica de estructuras de combustión vacías de restos, ni líticos ni faunísticos, en la planimetría de los niveles arqueológicos (Sañudo et al., 2012; Vallverdú i Poch et al., 2012; Vallverdú i Poch et al., 2010).

Una segunda aproximación metodológica a las estructuras de combustión del Abric es claramente más hermenéutica y basada en la analogía Etnoarqueológica y la Etnología prehistórica. La documentación del uso del fuego durante los trabajos de campo ha puesto de manifiesto la necesidad de precisar la descripción de la estructura de combustión en el registro arqueológico del Abric. El uso del fuego es singularizado por su técnica constructiva, en base a la observación de los hogares elementales que constituyen la estructura de combustión. La analogía con la distribución espacial de estructuras de combustión en cuevas, abrigos y asentamientos al aire libre documentada en las descripciones etnoarqueológicas y de la etnología prehistórica, es una vía de análisis para describir los asentamientos humanos exhumados en los niveles arqueológicos (Adouze, 1987; Arteaga et al., 2001a; Binford, 1988; Binford, 1996; Brooks y Yellen, 1987; Fisher y Strickland, 1991; Galanidou, 2000; Henry, 2012; Kent, 1991).

El análisis de las estructuras de combustión, y por extensión depósitos de ocupación musterienses del Abric, ha contado con una línea de trabajo fundamentada en disciplinas afines a la Geoarqueología, Arqueobotánica y Arqueometria. Estas investigaciones pueden ser integradas dentro de aproximaciones centradas en la Microarqueología con el fin de tratar de fundamentar el uso del espacio gracias a los restos pequeños (Mentzer, 2014; Weiner, 2010). Muchos de estos pequeños restos se consideran producto de actividades primarias ya que se acumulan en el lugar de su producción según los correlatos de la Arqueología del comportamiento (Schiffer, 1995). En el caso de registros arqueológicos susceptibles de ser formados por procesos culturales de mantenimiento, como ocurre en asentamientos de poblaciones con movilidad reducida (semisedentarios), los procesos de limpieza post hoc suelen provocar que los restos pequeños sean los únicos disponibles para la interpretación de funcional del registro y las estructuras arqueológicas documentadas (Mattews, 1994; Weiner, 2010).

Durante estos últimos 50 años de investigación arqueológica dedicada al análisis de las estructuras de combustión ha habido importantes avances en el análisis del registro microarqueológico. El análisis de las estructuras de combustión forma parte en realidad del continuo sobre la caracterización de los depósitos de ocupación. En ese sentido, la investigación de los sedimentos antrópicos, como una clase más dentro de las rocas sedimentarias, constituye una nueva vía de colaboración entre las ciencias de la Tierra y la Arqueología. En el siguiente apartado dedicado a los resultados vamos a describir sedimentos antrópicos, desde un punto de vista de las microfacies sedimentarias, con el fin de caracterizar las estructuras de combustión del nivel arqueológico $\mathrm{N}$ del Abric Romaní.

\section{Resultados}

\section{La estructura de los asentamientos prehistóricos del lecho arqueológico $\mathbf{N}$ del Abric Romaní}

El lecho del nivel $\mathrm{N}$ es un conjunto arqueológico constituido por pocos restos de fauna (200 huesos y dientes) y rocas talladas (unos 540 restos coordenados y 
un par de centenares de pequeños restos recuperados en los sedimentos lavados). En el nivel $\mathrm{N}$ también ha sido documentada una impronta de madera de $5.1 \mathrm{~m}$ de largo por $0.06 \mathrm{~m}$ de ancho sin ramas (Fig. 3). El número de estructuras de combustión es 19 (Fig. 4) (Vallverdú i Poch et al., 2010). La distribución espacial con un metro de separación, documentada en un grupo de estructuras de combustión del lecho N (Fig. 3), facilitó una analogía etnológica con las áreas dormitorio documentadas por la Etnoarqueología y la Arqueología prehistórica (Binford, 1988; Binford, 1996; Movius, 1966). Estas áreas de actividad, como ocurre con la documentada en el nivel $\mathrm{N}$ del Abric Romaní, suelen estar desprovistas de restos de rocas talladas y de fauna. Estas áreas son conocidas también como áreas de actividad de descanso y reposo, y son consideradas uno de los componentes básicos de la estructura de los yacimientos / asentamientos residenciales en las observaciones etnoarqueológicas (Binford, 1987; Binford, 1988)

El área de actividad de dormitorio del nivel $\mathrm{N}$ se documentó en la que se considera la zona interna del asentamiento, pegada a la pared noroeste del abrigo
(Vallverdú i Poch et al., 2010). En la zona externa pudimos describir otras estructuras de combustión con restos de fauna y rocas talladas que en su mayoría son sobre sílex. El área dormitorio del nivel $\mathrm{N}$ está fechado entorno 54 ka BP y su interpretación como tal, apunta a una primera aparición de asentamientos residenciales de poblaciones humanas arcaicas en el registro arqueológico mundial.

Las estructuras de combustión del lecho $\mathrm{N}$ fueron documentadas en la planimetría de campo de forma individualizada mediante el mismo protocolo (Fig. 5) (Vallverdú i Poch et al., 2012). También fueron muestreados los sedimentos carbonosos, los sedimentos enrojecidos por la rubefacción y los sedimentos naturales subyacentes de cada estructura de combustión a medida que se exhuma la estructura. Durante la elaboración de la sección microestratigráfica de la estructura de combustión se aprovecha el perfil para obtener muestras sin perturbar para ser posteriormente incluidas en resinas poliéster en el laboratorio. Las muestras polimerizadas se cortan para fabricar láminas delgadas de gran tamaño $(14 \times 6 \mathrm{~cm})$ con el grosor estandarizado para el análisis petrográfico.

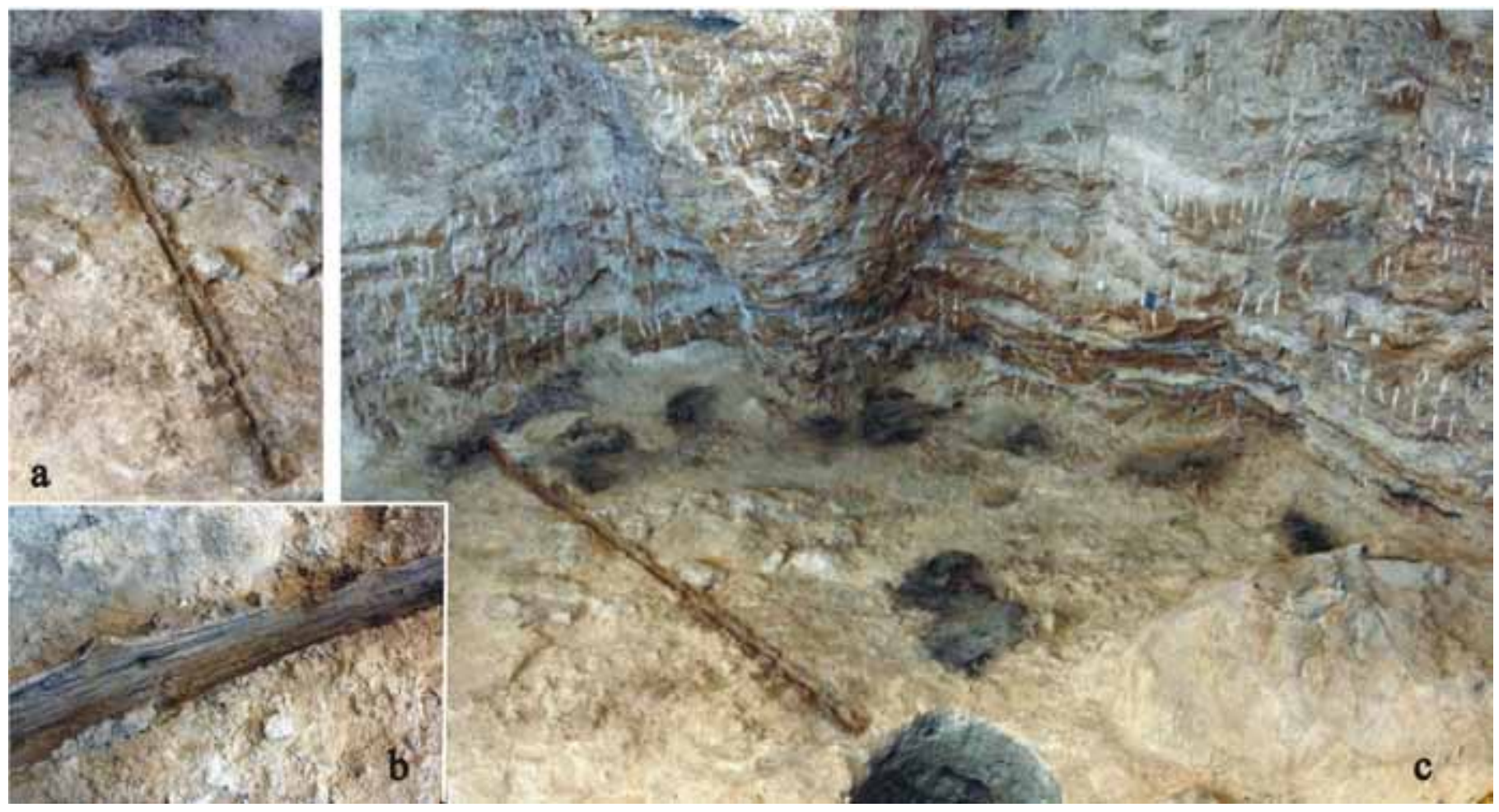

Figura 3. Fotografías de la impronta de madera (a), una vista detallada de la impronta de la madera en el travertino (b), y una vista general de las estructuras de combustión de la zona interna del nivel $\mathrm{N}$ del Abric Romaní (Capellades, provincia de Barcelona, Spain) (c).

Figure 3. Photographs of the wood imprint (a), a detailed view of the wood imprint in the travertine (b), and a general shot of the combustion structures in the inner zone of archaeological bed $N$ at the Abric Romani rock-shelter (Capellades, province of Barcelona, Spain) (c). 


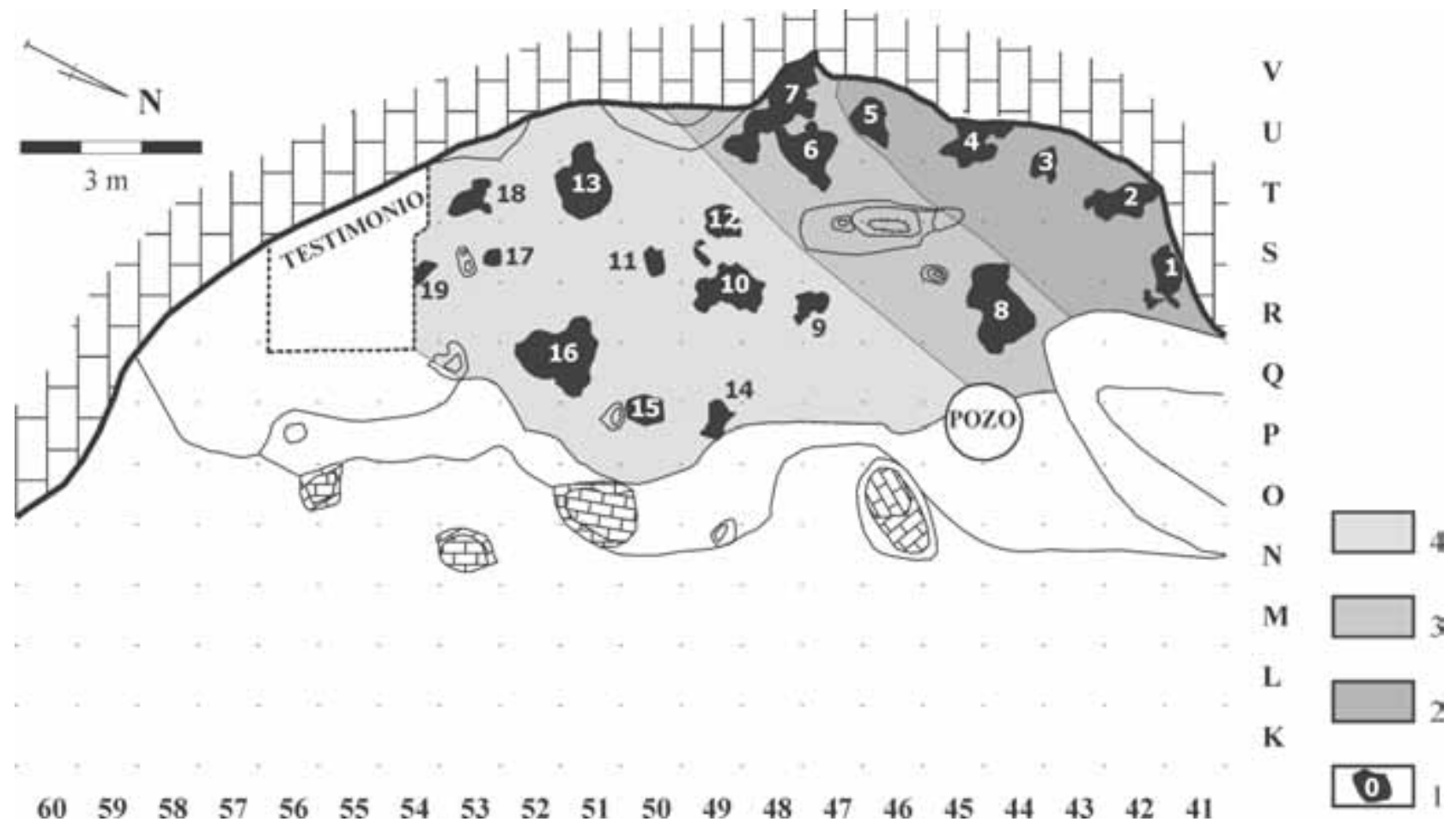

Figura 4. La planimetría de la superficie del nivel $\mathrm{N}$ con la posición espacial y número de las estructuras de combustión (1), y las áreas que ocupan la zona externa (2), intermedia (3) e interna (4) del asentamiento prehistórico fechado en torno a los 54 ka B.P. (Vallverdú i Poch et al., 2010).

Figure 4. The cartography of the surface of archaeological bed $N$ with the spatial location and number of the combustion structures (1), and the area of the outer (2), intermediate (3) and inner zones of the prehistoric settlement dating to 54 ky B.P. (Vallverdú $i$ Poch et al., 2010).

\section{Microfacies sedimentarias}

Las observaciones microscópicas de las muestras de las estructuras de combustión del nivel $\mathrm{N}$ del Abric están constituidas por dos microfacies: gravas y arenas grises, y gravas con limo-arena gris marrón. Esta clasificación se basa en la intensidad de alteración de los fragmentos de travertino y los rasgos edáficos calcíticos de los sedimentos expresada por su coloración (Vallverdú i Poch and Courty, 2012). La intensidad de alteración fuerte es expresada por la coloración gris y gris marrón. Estas microfacies contienen incorporaciones texturales y modificaciones por la antropización de los sedimentos. Las de coloración gris y gris marrón presentan rasgos edáficos y son descritas mediante la micromorfología de suelos (Bullock et al., 1985; Stoops, 2003).

Las gravas grises presentan vacuolas de disolución y micritización. Es frecuente la pérdida del contorno de las gravas en contacto con el sedimento encajante. La fracción fina es rara, la distribución relativa gefúrica y la microestructura vesicular y laminar. Estos fragmentos de roca muestran revestimientos calcíticos y los limos-arenas están microagregados en formas granulares finas micritizadas de contorno rugoso. Los rasgos texturales son raros a pocos y se reducen a rellenos sueltos discontinuos y cúpulas enlazadas. Las microfacies grises muestran pocos a raros microrrestos antrópicos y transformaciones de color rojizo. El enrojecimiento es atribuido a la rubefacción y conforme a la mejora cristalina de los óxidos de hierro criptocristalinos del material parental (Canti y Linford, 2000).

Las microfacies gris marrón muestran abundantes adiciones de microrrestos antrópicos. Las gravas tienen un contorno irregular y liso con pocos revestimientos calcíticos. Los limos y las arenas son relativamente escasos y su microestructura es compleja, sin agregación y constituida por una microestructura de empaquetamiento simple y otra de cavidades. Algunos limos muestran un color marrón amarillo dispuesto sobre la porosidad de cavidades, o en forma de bandas separadas por la porosidad vesicular. La distribución relativa es chitónica a gefúrica. Los rasgos edáficos son texturales y formados por rellenos sueltos discontinuos. Los rasgos criptocristalinos 
52

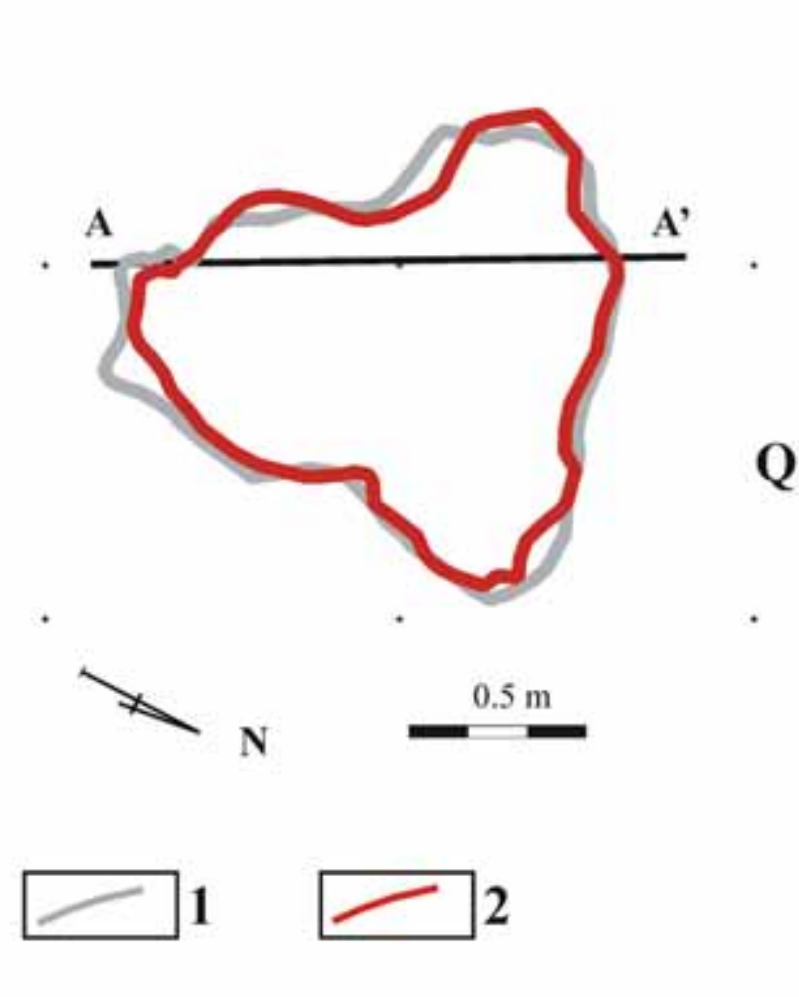

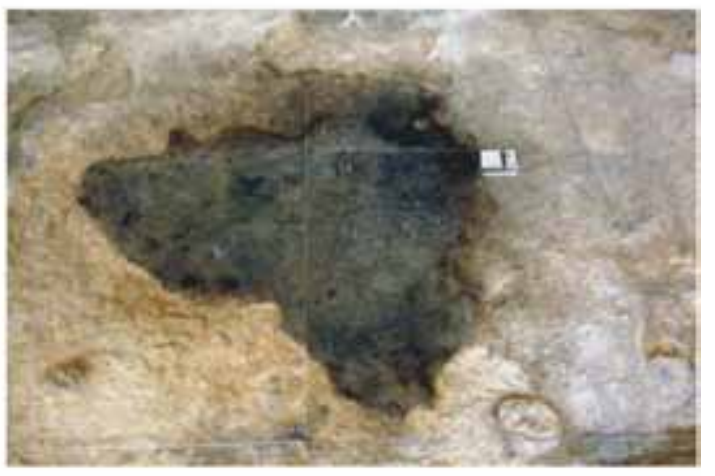

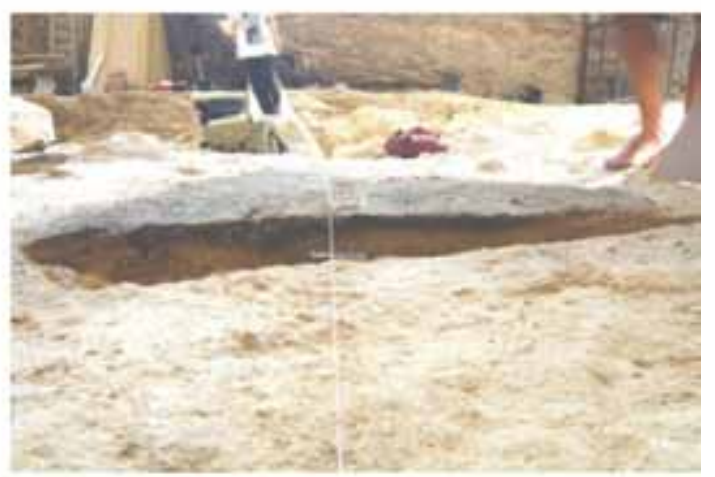

A

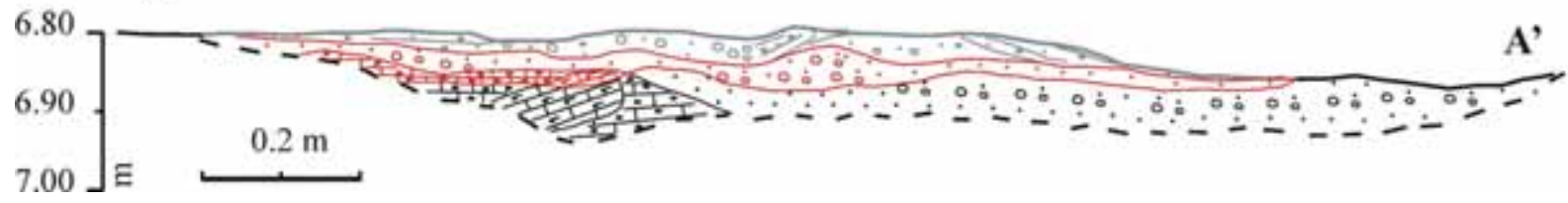

Figura 5. Documentación de campo de la estructura de combustión 16 del nivel arqueológico $\mathrm{N}$ basada en la cartografía y la microestratigrafía. La planta representa el contorno de la extensión de los depósitos ricos en carbón vegetal (carbonosos) (1) y enrojecidos (rubefactados) (2) de la estructura de combustión 16. A la derecha de la ilustración fotografías de la planta y la sección de la estructura de combustión 16. Debajo, la microestratigrafía del perfil señalado en la planta (A-A'). En el perfil hay representadas los depósitos carbonosos (en gris pálido), enrojecidos (en gris oscuro) y naturales (en negro). El espesor representado es la referencia sobre el punto cero del yacimiento.

Figure 5. Field record of combustion structure 16 of archaeological bed $N$ based on cartography and microstratigraphy. The frontal view schematic shows the contour of the plant-charcoal-rich (carbonaceous) (1) and reddened (2) deposits of combustion structure 16. Below, the microstratigraphy of profile $\left(A-A^{\prime}\right)$ set in the image above. The profile shows the plant-charcoal-rich (carbonaceous) (in pale grey), reddened (in dark grey) and natural (in black) deposits. The thickness refers to the zero level of the archaeological site.

tienen relación con la micritización marrón amarilla de pequeñas masas calcíticas de poca densidad

\section{Microfacies antrópicas de las estructuras de combustión del nivel $N$}

Las microfacies de las estructuras de combustión forman parte de un continuo dentro de los depósitos que contienen los conjuntos de restos arqueológicos y han sido tratadas de forma más sistemática dentro de las microfacies antrópicas del nivel $\mathrm{J}$ del Abric (Vallverdú i Poch and Courty, 2012). Las modificaciones térmicas se reconocen a través de la fragmentación y el cambio de color (Wattez, 1988; Wattez, 1990). En las láminas delgadas también han sido determinadas otras modificaciones antrópicas como la compactación y las modificaciones naturales producidas por la crioturbación (Figs. 6.1, 6.2 y 6.3).

Los travertinos afectados por el calor presentan un 
aumento de la red de fisuras y liberación de constituyentes, opacificación de las micritizaciones, abundancia de microesparitas angulares de alta birrefringencia. También han sido descritas microesparitas agregadas microgranulares en masas continuas y en empaquetamiento suelto. Por otro lado, los fragmentos de travertino calcinados muestran una fábrica interna blanca criptocristalina de calcita de muy alta birrefringencia. Algunas de estas masas criptocristalinas contienen microcarbones y pueden ser consideradas pseudomorfos de calcita de oxalatos de calcio (cenizas) añadidos a los sedimentos travertínicos (Fig. 6.3) (Brochier and Thinon, 2003; Canti, 2003; Courty, 1984; Wattez, 1988).

Hay otros cambios en la fábrica interna de los fragmentos de travertino quemados al poseer colores variables: coloración marrón amarilla; coloración gris; masas criptocristalinas blancas puras y amariIlentas. Estas fábricas argumentan distintos estados de temperaturas y aireación que sugieren la mezcla de transformaciones térmicas características de estructuras de combustión con más de un episodio de uso del fuego. Las masas criptocristalinas puras amarillentas son consistentes con la exposición subaérea por corrosión detectada también en las microfacies de color gris marrón (Figs. 6.4 y 6.6). La corrosión de estas masas criptocristalinas amarillentas fundamenta la interpretación según la cual el uso del fuego se produce de forma interrumpida en el tiempo, pero de forma congruente en la misma estructura de combustión.
Los residuos de la combustión documentados durante los trabajos de campo en el nivel $\mathrm{N}$ son descritos por su dominante contenido en microcarbones. La composición carbonosa de estos residuos apunta a un uso del fuego en el que se alcanzan temperaturas bajas. Esta observación concuerda con el tamaño pequeño $(<0.2 \mathrm{~m})$ de los lechos lenticulares carbonosos medidos en la microestratigrafía de las estructuras de combustión (Vallverdú i Poch et al., 2012; Vallverdú i Poch et al., 2010). Sin embargo, las microfacies antrópicas ilustran sobre el contenido calcítico derivado de la fragmentación de las rocas travertínicas por el calor y las adiciones en forma de ceniza de los combustibles vegetales usados como combustibles (Fig. 6.6). En este sentido, se observa a simple la vista o con el microscopio petrográfico un bajo porcentaje de los fragmentos de carbón vegetal en las láminas delgadas (Fig. 7.5). De todas formas, es necesario interpretar la integridad de los residuos de combustión a la luz de los procesos de calcificación descritos en las microfacies de gravas y limo arena gris y gris marrón. Así, la intensidad de alteración fuerte atribuida a las microfacies grises en principio ha de favorecer su conservación a través de la calcificación y expresada por las calcitas pseudomórficas de las cenizas y exhibido de manera más evidente por la abundancia de fragmentos de travertino calcinados. La corrosión observada en las microfacies marrón gris, coherente con la decalcificación, no es acompañada por abundantes rasgos edáficos calcíticos (Fig. 6.6). Por tanto, las microfacies de grava y limo-arena

Figura 6. Imágenes y microfotos de una lámina delgada muestreada de la estructura de combustión 16 en la zona externa del modelo de asentamiento del nivel $\mathrm{N}$ del Abric Romaní (Fig. 4). 1, imagen de la lámina delgada en la que se muestra sus figuras sedimentarias principales y la posición de las microfotos de la figura 6. 2, imagen escaneada ampliada de una costra sedimentaria, en la mitad inferior, en el que existe una porosidad fisural horizontal y partículas de sedimentarias con un empaquetamiento denso. 3, microfoto de microesparitas empaquetadas en masas granulares. Estas masas granulares son de color gris claro y gris opaco oscuro. El fragmento pseudomórfico microsparítico oscuro es ceniza calcificada opaca. 4, masas granulares microsparíticas grises y gris amarillo con fragmentos de travertinos opacos. La alteración de las masas granulares microsparíticas, señalada por el color gris amarillento, apunta a la aparición de la corrosión. 5, microestructura vesicular en las microfacies de grava y arena gris, con rasgos texturales en casquetes continuos (link cappings) opacos (con fábrica de birrefringencia indiferenciada) que apuntan al enrojecimiento criptocristalino (rubefacción) (Canti y Linford, 2000). 6, microestructura vesicular en las microfacies de gravas y limo-arena gris marrón. Estas microfacies muestran un empaquetamiento denso y una fisuración subhorizontal. 7, fragmento pseudomorfico de hoja vegetal con esferulitos drusos criptocristalinos típicos (Brochier, 1983b). 8, microcarbón rodeado por microsparitas de color marrón amarillas relacionadas con la temprana calcificación pseudomórfica de oxalatos de calcio de cenizas.

Figure 6. Images and microphotos from a thin section sampled from combustion structure 16 at the outer zone of the Abric Romani level $N$ settlement pattern. 1, scanned image of the thin section showing sedimentary figures and the position of the microphotos of figure 6. 2 , enlarged scanned image of a sedimentary crust, in the lower half of the image, with horizontal fissural porosity and sedimentary particles with dense packing. 3, microphoto of microsparites packed in granular masses. These granular masses are pale and dark grey. The dark grey pseudomorphic microsparitic fragment is an opaque calcified ash fragment. 4, microsparites in grey and brownish-grey granular masses with opaque travertine rock fragments. The weathering of the granular microsparites, evidenced by their yellowish-grey color, suggests the appearance of corrosion. 5, vesicular microstructure in the grey gravel and sand microfacies. The microphoto shows textural features made from opaque link cappings (with undifferentiated b-fabric) pointing to cryptocrystalline reddening (rubefaction) (Canti and Linford, 2000). 6, vesicular microstructure in brownish-grey gravels and sandy silt microfacies. These microfacies appear with dense packing and horizontal fissures. 7, pseudomorphic fragment of a plant leaf showing typical cryptocrystalline druse spherulites (Brochier, 1983b). 8, microcharcoal surrounded by yellowish-brown microsparite related to the early calcitic pseudomorphose of the calcium oxalates of ashes. 

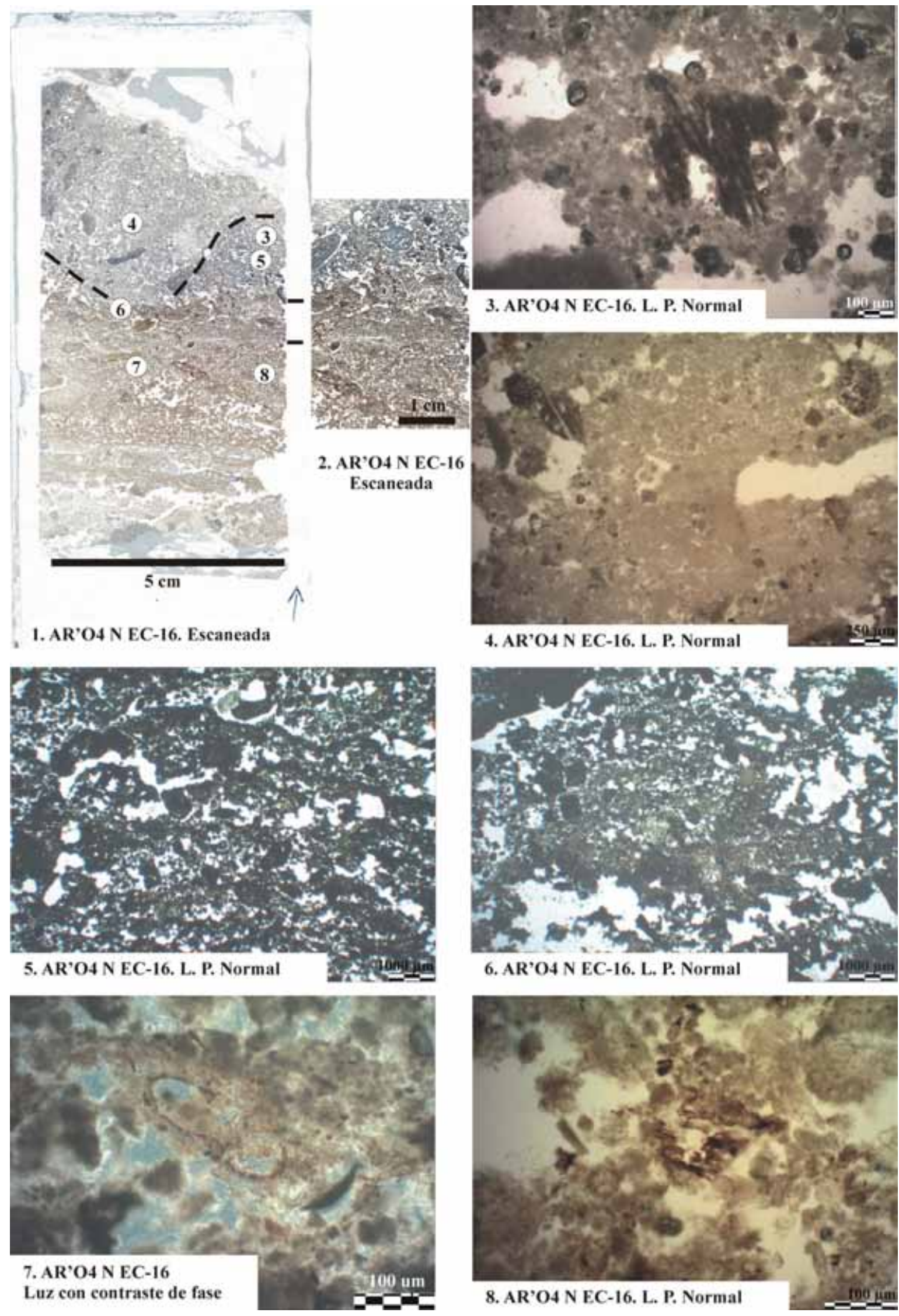
gris marrón sugieren un rápido enterramiento, por fragmentos de roca con la alteración fuerte heredada de la pared del abrigo. La microestructura compuesta no agregada, descrita por el empaquetamiento simple y la porosidad de cavidades, también apunta al enterramiento rápido de las microfacies gris marrón que contienen gran parte de los microrrestos antrópicos (Figs.7.6 y 7.7).

La calcificación de las microfacies de grava con arena-limo gris tiene por origen la humedad y la evapotranspiración provocada por el goteo de agua bicarbonatada de la visera del abrigo. Como ejemplo, en la figura 6.1 detallamos dentro de la imagen de la lámina escaneada una figura sedimentaria de corte y relleno con un trazo continuo de forma cóncava. Estas figuras sedimentarias ya fueron observadas en las descripciones de campo y la observación en la lámina delgada. La geometría de este depósito y su relleno sugiere la transformación de los componentes quemados por el goteo de agua. El microambiente húmedo también está de acuerdo con la presencia de restos de hojas y esferulitos drusos de carbonato de calcio reseñados de forma común en depósitos de medio húmedos, y ausentes en depósitos de medios secos (Fig. 6.7) (Brochier, 1996). La microestructura laminar y vesicular (piprakes) (Fig. 6.5), muestra la incidencia de la crioturbación superficial (periglaciar). Estos rasgos micromorfológicos criogénicos argumentan la incidencia de la desecación (evapotranspiración) en el desarrollo de los rasgos edáficos calcíticos de las microfacies gris del nivel arqueológico $\mathrm{N}$.

Las láminas delgadas escaneadas de las figuras 7.1 y 7.5 pertenecen a estructuras de combustión de la zona interna (EC 4 y 7 Fig. 4). La figura 6.1 pertenece a otra estructura de combustión, pero de la zona externa (EC 16, Fig. 4). Una de las características microestratigráficas observada en el campo fue la diferencia en el grosor de los sedimentos carbonosos y rubefactados en los perfiles de las estructuras de combustión de la zona interna respecto a las de la zona externa (Vallverdú i Poch et al., 2010). Las diferencias fueron atribuidas, a pesar de ser en un número limitado de observaciones, a una clase distinta de talla $\left(e^{2} \mathrm{~m}^{2}\right.$ ) de estructuras de combustión. Esta diferencia en la talla fue atribuida a tamaños distintos de los hogares elementales, además de un distinto patrón de solapamiento de las terminaciones de los lechos lenticulares carbonosos de las estructuras de combustión (Vallverdú i Poch et al., 2010): para el uso del fuego en la zona interna, dominaba la acreción vertical de hogares elementales, mientras que en la zona externa, las estructuras de combustión podían haber sido formadas por la acreción lateral de los hogares elementales.

Las láminas delgadas escaneadas de la EC 7, de la zona interna, respecto a la muestra de la EC 16, de la zona externa (Fig. 4), permiten observar las diferencias en el grosor de las facies carbonosas (Figs. $6.1 \mathrm{y}$ 7.5). La observación microscópica es complementaria y también contradictoria. Especialmente con la observación de campo, en cuanto los grosores de las facies carbonosas. Una de las muestras para las observaciones microscópicas, en particular la de la estructura de combustión 7 (Fig. 7.5), ilustra el pequeño grosor de las acumulaciones carbonosas en la zona interna y alerta del salto de escala de observación para estimar la composición del lecho carbonoso cuando es descrita en el campo o en lámina delgada. Ya hemos descrito cómo la observación de la lámina delgada permite estimar la cantidad y la integridad de registro sedimentario de los residuos de combustión. Y también contrastar la heterogeneidad de los fragmentos de rocas quemados. Sin embargo, la muestra de la estructura de combustión 4 (Fig. 7.1) de la zona interna contiene acusadas transformaciones debidas a la calcificación (Figs. 7.3 y 7.4) y la no preservación por

Figura 7. Imágenes y microfotos de láminas delgadas de las estructuras de combustión 4 y 7 de la zona interna del modelo de asentamiento del nivel N. 1, imagen escaneada de la lámina delgada de la EC 4 en la que se ve la gradación y el empaquetamiento denso de los lechos con las microfacies de gravas y arenas grises. 2, detalle ampliado de un lecho compacto en el centro de la imagen donde se observan, en las partículas del tamaño arena gruesa y gránulo, componentes quemados con distinta intensidad y reproducidas con un color gris más oscuro. 3 y 4 , microfoto de la calitización sobre componentes cristalíticos quemados que muestran una birrefringencia más alta que la calcita no quemada. 5, lámina delgada escaneada de una muestra de la EC 7 en el que se observa el pequeño tamaño del lecho carbonoso y fragmentos de travertino quemados con distinta intensidad indicado por un color más gris. 6 y 7 , componentes sedimentarios de la estructura de combustión 7 añadidos. En este caso, agregados criptocristalinos rojizos con partículas siliclasticas atribuidos a combustibles leñosos (madera y corteza) (Weiner, 2010).

Figure 7. Thin section scanned image and microphotos from combustion structures 4 and 7 of the inner zone of the level $N$ settlement pattern. 1, scanned image of the combustion structure 4 thin section showing beds with graded and dense packed grey microfacies. 2 , improved scanned detail of the thin section focusing on the laminae with dense packing of the particles at the center of the image. The grey coarse sand and granule sized material includes sedimentary particles burned to differing intensities, indicated with darker grey. 3 and 4, microphoto of the calcification on burned crystalline components showing higher birefringence colors than non-burned calcite. 5, scanned thin section from the combustion structure 7 sample showing the small size of the charcoal-rich bed and travertine rock fragments burned at different intensities. 6 and 7 , sedimentary components added to combustion structure 7 . In this case, red cryptocrystalline aggregate with siliciclastic particles are related to wood and bark fuel (Weiner, 2010). 

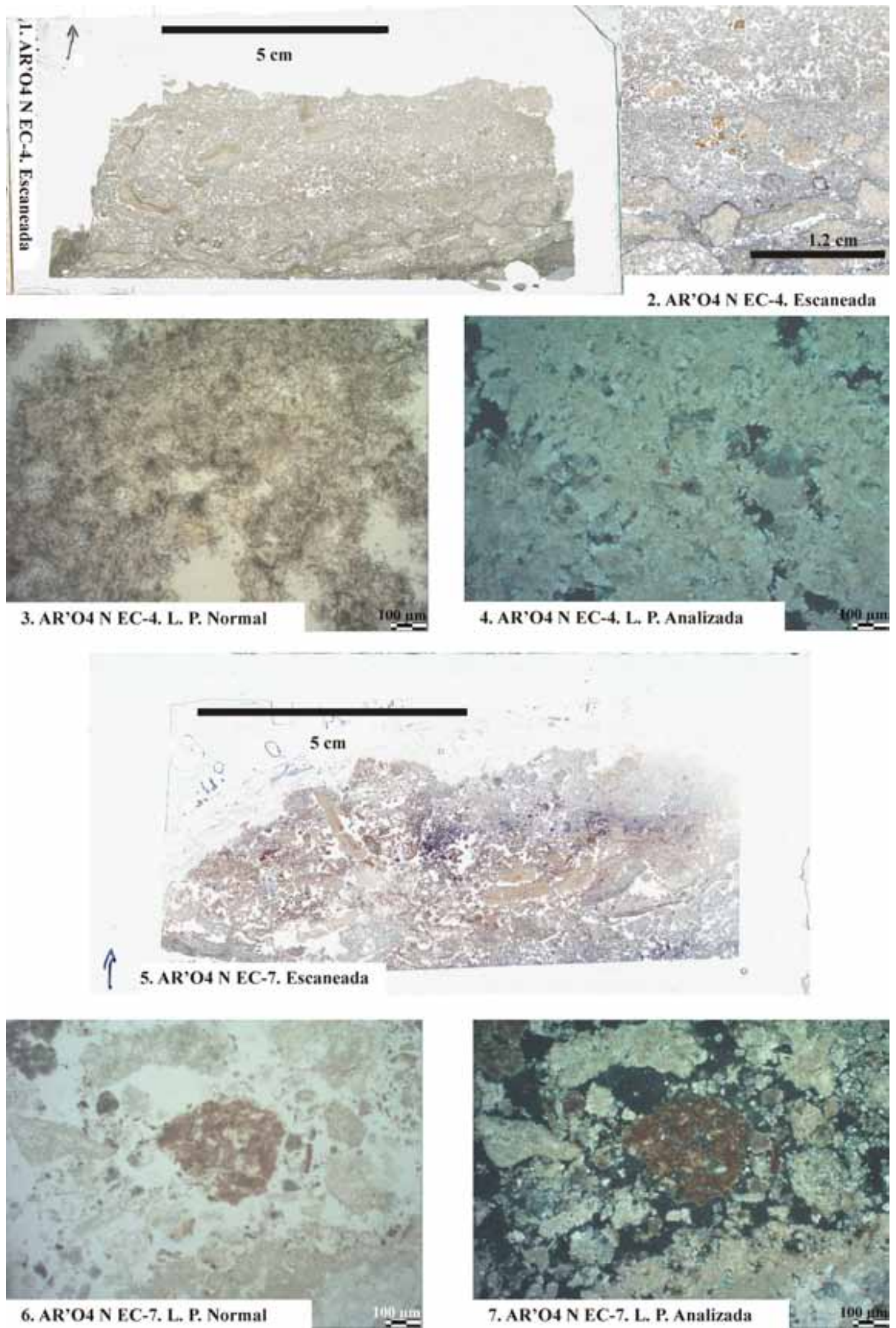
erosión del lecho carbonoso. La evidencia de la rubefacción, preservada por la calcificación en las microfacies sedimentarias de gravas y limo arena gris, alerta sobre las condiciones locales (azonales) de preservación del lecho carbonoso y la evolución previa al enterramiento. En este caso, la estructura de combustión 4 (Fig. 7.1) está truncada por la escorrentía superficial. La existencia de la estructura sedimentaria gradada, observada en la lámina delgada y formada con anterioridad al impacto térmico antrópico (Figs. 7.1 y 7.2), permite plantear que esta muestra de la estructura de combustión 4 ilustra una posible interacción entre los procesos de formación naturales y culturales en el registro sedimentario. Así, es posible argumentar que la estructura sedimentaria gradada tenga que ver con la compactación. La compactación se acompaña de un consecuente cierre de la porosidad estructural y cese de la percolación vertical para favorecer la escorrentía superficial. En el caso de los sedimentos antrópicos, la compactación por pisoteo es a menudo descrita en los depósitos de ocupación fosiles y experimentales mediante la micromorfología de suelos. La descripción de la compactación por pisoteo en los depósitos de ocupación se apoya en la guía de las investigaciones realizadas en torno a la formación de costras sedimentarias generadas por el impacto de las gotas de la lluvia y su relación con las investigaciones realizadas sobre los procesos de degradación de la superficie de los suelos (Gé et al., 1993; Goldberg and Whitbread, 1993; Miedema, 1997).

\section{Discusión}

Las descripciones y los comentarios petrográficos y micromorfológicos, dedicados a las muestras de láminas delgadas de las estructuras de combustión del nivel N, han intentado situar las observaciones microscópicas a continuación de las observaciones microestratigráficas de campo para evitar saltos de escala. Hemos visto por ejemplo que las descripciones litológicas de los depósitos ricos en microcarbón en el campo no tienen nada que ver con las de las microfacies antrópicas constituidas principalmente por rocas quemadas y pocos carbones. $Y$ hemos advertido que medidas de campo microestratigráficas, como el grosor de los depósitos carbonosos, pueden ser confirmados por algunas de las muestras y por otras no. Así, en las estructuras de combustión del nivel $\mathrm{N}$ existen procesos postsedimentarios, como es el caso de estructuras de combustión afectadas por la compactación y la escorrentía superficial, o las figuras de corte y relleno asociadas al goteo de agua, que pueden explicar diferentes fenómenos de distinto orden para la interpretación paleoambiental y arqueológica del nivel $\mathrm{N}$.

La continuidad entre escalas de observación de las estructuras de combustión del nivel $\mathrm{N}$ confirma que el uso del fuego transforma y forma registro sedimentario en el nivel N. En la investigación arqueológica de depósitos de ocupación humana es común el interés en confirmar el uso del fuego en relación a la composición y distribución espacial (huesos quemado y no quemados, cenizas, rocas talladas quemadas, etc.) (Goren-Inbar et al. 2004; Weiner et al., 1988). Estas investigaciones están formalizadas en yacimientos en los que existen importantes sesgos en la integridad de los depósitos que contienen apenas rasgos de combustión antrópica (Mentzer, 2014). EI análisis y descripción de los rasgos de combustión focaliza en parte la investigación geoarqueológica, tal como ocurre por ejemplo en la reciente discusión sobre la generalización del uso del fuego por las poblaciones humanas arcaicas y sus implicaciones evolutivas sobre Homo (Roebroeks y Villa, 2011; Sandgathe et al., 2011). Sin embargo, la preservación de las estructuras de combustión en el registro sedimentario de los yacimientos arqueológicos son aún en parte mal conocidas. En los yacimientos de poblaciones arcaicas del mediterráneo oriental, el uso del fuego ha sido muy fundamentado, y de manera especial en niveles arqueológicos con sedimentos antrópicos de gran espesor, como por ejemplo en la cueva de Kebara (Goldberg et al., 1994; Meignen et al., 2001; Shahack-Gross, 2008; Speth, 2006). En estos yacimientos los sedimentos antrópicos son dominantes en sus registros sedimentarios, de forma análoga a los yacimientos de cueva redil holocenos como los documentados en el sureste de Francia (Brochier, 1983a; Brochier, 1983b; Brochier, 1996; Courty, 1984).

Las estructuras de combustión del Abric realizadas por poblaciones humanas arcaicas hasta ahora han sido muy singulares en el registro arqueológico europeo (Rigaud et al., 1995). Sin embargo, la exploración y revisión de yacimientos arqueológicos de poblaciones arcaicas, gracias a nuevos proyectos de investigación, ha aumentado las evidencias de los rasgos de combustión en el registro sedimentario pleistoceno, y en especial en el de la Península Ibérica (Sala, 2014).

La explotación arqueológica de estos testimonios del uso del fuego no tiene sólo implicaciones evolutivas para las poblaciones de homo. En el marco de la investigación de los depósitos de ocupación, esbozado más arriba en el apartado de metodología, el registro sedimentario del uso del fuego ofrece una línea de investigación poco explorada sobre su distribución espacial y el uso del espacio. Y los resultados del Abric, a partir de la intervención arqueológica pla- 
nimétrica, nos han permitido documentar e interpretar el registro arqueológico con un objetivo distinto al de las excavaciones estratigráficas o las basadas en confirmar el uso del fuego (Coudart, 2002; Schiffer, 1987a; Stein and Rapp, 1985).

La investigación de la estructura de los asentamientos de las poblaciones humanas arcaicas en los niveles arqueológicos de Abric trata de determinar los aspectos que $L$. Binford señaló en su definición de estructura de un yacimiento / asentamiento (Binford, 1987; Binford, 1988): el tamaño del área del asentamiento, la determinación y disposición de las áreas de actividad y sus construcciones (diferentes tipos de hogares, muros, apilamientos y enlosados de rocas, etc.). En este sentido, la Geoarqueología no sólo asiste a la documentación de la intervención arqueológica con la preocupación de respetar la estratificación si no también la de cartografiar y caracterizar las áreas de actividad y las construcciones del asentamiento. Así, el estudio de los sedimentos antrópicos, planteado en la planimetría de las superficies excavadas en los yacimientos arqueológicos, puede caracterizar mapas de litofacies antrópicas en base a muestreos sobre estas superficies. Estas investigaciones geoarqueológicas más recientes han documentado sedimentos antrópicos de referencia para caracterizar otros tipos de depósitos de ocupación (Adderley et al., 2010; Courty et al., 1989; Courty et al., 1994; Goldberg and Macphail, 2010; Goldberg and Mcphail, 2006; Goldberg et al., 1993; Mattews, 1994). Estos referenciales se basan principalmente en componentes (orgánicos y minerales), y de forma notable, microestructuras de suelos.

La preservación de las estructuras de combustión del Abric Romaní obedece al uso repetido y congruente del fuego en la superficie del asentamiento. En definitiva, las estructuras de combustión llegan a ser visibles en los palimpsestos arqueológicos por tratarse de áreas de actividad donde el uso del fuego es repetido durante uno o más episodios inter o intra ocupacionales, en la misma área de actividad. En cierto modo, de forma análoga ocurre con las litofacies, en las que hay subyacente la naturaleza temporal del concepto lámina / lecho (formadas por uno o más eventos deposicionales) (Campbell, 1967). Las investigaciones etnoarqueológicas también advierten que las actividades asociadas a una redundancia espacial (eventos ocupacionales múltiples en la misma superficie) son sobrerepresentadas en el registro arqueológico. El uso redundante del espacio regional y local intensifica el potencial para identificar áreas de actividad (Brooks and Yellen, 1987).

La descripción de un asentamiento residencial en el nivel $\mathrm{N}$ no cuadra mucho con las observaciones etnoarqueológicas (Brooks and Yellen, 1987). Los asentamientos de unidades domésticas residenciales están poco representados en el registro arqueológico. Las observaciones etnoarqueológicas argumentan que los grupos humanos evitan el uso repetido de una misma superficie para establecer los asentamientos residenciales. Por ejemplo, los !Kung evitan reutilizar campamentos residenciales hasta que sus evidencias en la superficie del terreno desaparecen (Brooks and Yellen, 1987). Esta práctica parece ser ventajosa: evita la sobreexplotación de alimentos y leña, exposición a las enfermedades, insectos y predadores. Aunque también la localización espacial de recursos estratégicos en el paisaje, como el agua, puede condicionar el uso repetido de asentamientos residenciales. Es significativo que la dispersión espacial de los asentamientos residenciales, fundamentada en una limitada utilización repetida de la localidad de asentamiento, provoca su invisibilidad arqueológica en el registro pleistoceno surafricano (Brooks and Yellen, 1987).

La visibilidad arqueológica del área dormitorio del nivel $\mathrm{N}$ se fundamentada por la analogía etnoarqueológica y el registro arqueológico paleolítico (Vallverdú i Poch et al., 2010). El uso del actualismo causal en la analogía etnográfica, y en general la linealidad en el razonamiento hipotético deductivo, ha sido ciertamente criticado por reduccionista y trivial (Coudart, 2002). Sin embargo, consideramos que la analogía es una guía para tratar de interrelacionar los modelos teóricos antropológicos - etnológicos y las reconstrucciones empíricas del registro material arqueológico.

En la figura 8 representamos los datos de campo relativos a lo que parece ser, en base a la escala de observaciones microestratigráficas y planimétricas, una zona de dormitorio con baja visibilidad arqueológica en el nivel O. Esta zona contiene diversas estructuras de combustión y, junto a los restos coordenados, ha sido denominada zona $\mathrm{O} 10$ en un estudio interdisciplinar llevado a cabo recientemente (Chacón et al., 2015). La visibilidad arqueológica de las estructuras de combustión de la zona $\mathrm{O} 10$ no es tan válida para la analogía como las estructuras de combustión de la zona de reposo citada del nivel $\mathrm{N}$. El análisis de los restos registrados en la zona $\mathrm{O} 10$ identifica episodios de actividad individuales mediante remontajes de fauna y rocas talladas que conectan con otras zonas de actividades sincrónicas y diacrónicas. Además, argumentan otros episodios de actividad diacrónicos en el conjunto arqueológico $\mathrm{O}$ mediante la proyección de las coordenadas tridimensionales en perfiles.

La zona 010 es definida por ser un área de activi- 
dad doméstica ya que contiene rocas talladas y huesos con marcas de corte acumulados alrededor de hogares (Chacón et al., 2015). En los restos de fauna, localizados en la base del nivel arqueológico, destacan huesos afectados por las Ilamas. Este dato proporciona la evidencia para establecer la posibilidad de argumentar actividades de limpieza de restos óseos ya consumidos, o la utilización de huesos como combustible de las hogueras. La microestratigrafía de esta estructura de combustión indica la presencia de lechos carbonosos grises (heterogéneos, con rocas quemadas y sin quemar) que apuntan a la dispersión (depósitos secundarios) de los depósitos carbonosos (Vallverdú i Poch et al., 2012). La formación de acumulaciones terciarias (Butzer, 1989), a partir de depósitos carbonosos de uso del fuego o también conocidas como depósitos de limpieza / vaciado de hogares (Wattez, 1990), no están presentes en el registro sedimentario antrópico de la zona 010.

La zona de actividad de $\mathrm{O} 10$ es considerada un palimpsesto difícil de caracterizar mediante los restos culturales (Chacón et al., 2015). Sin embargo, con la ayuda del conjunto arqueológico estudiado se caracterizan distintos episodios de actividad para ilustrar, al menos un número mínimo de eventos ocupacionales sincrónicos y diacrónicos, una dimensión temporal análoga al evento etnográfico. Las estructuras de combustión de en esta zona $\mathrm{O} 10$ muestran una distribución espacial y una microestratigrafia caracterizada por representar también distintos eventos de uso del fuego (microestratificados), y por tanto con una sincronía difícil de demostrar.

Las observaciones y medidas obtenidas de la planimetría de la zona 010 muestran una estructura de combustión de gran tamaño de $7 \mathrm{~m}^{2}$ y otras más pequeñas de área centimétrica, algunas de ellas separadas por intervalos decimétricos (Chacón et al., 2015). Todas las estructuras de combustión de la zona O10 se localizan entre 0.5 y $3 \mathrm{~m}$ de la pared. La estruc- tura de combustión de gran tamaño tiene una forma rectangular, contiene travertinos quemados y una pendiente medida en el eje mayor del $4 \%$. La microestratigrafía de esta estructura de combustión mayor se ha realizado construyendo diferentes perfiles ilustrados en la figura 8. La observación microestratigráfica en los tres perfiles ha permitido medir, siguiendo el protocolo de exhumación de las estructuras de combustión del Abric Romaní (Vallverdú i Poch et al., 2012), 15 lechos lenticulares compuestos por facies carbonosas de $20 \mathrm{~cm}$ de longitud con un grosor máximo de $3 \mathrm{~cm}$; el grosor máximo medido en 8 estratos rubefactados es de $3 \mathrm{~cm}$; se han determinado hasta 3 lechos lenticulares con facies carbonosas grises heterogéneas (con rocas quemadas y no quemadas) con una longitud y grosor máximos de $60 \mathrm{~cm}$ y $10 \mathrm{~cm}$ respectivamente. En las secciones microestratigráficas se observan lechos lenticulares carbonosos sobrepuestos a lechos rubefactados imbricados (Fig. 8, sección $\left.A-A^{\prime}\right)$. Algunos de estos lechos carbonosos con la suela de rubefacción forman grupos separados por distancias inferiores a 1 metro (Fig. 8, sección B - B').

La medida del tamaño y grosor máximos de los lechos lenticulares que contienen las facies carbonosas es significativa al ilustrar una aproximación al tamaño decimétrico $(0.2 \mathrm{~m})$ de los hogares elementales. Estos hogares elementales pequeños han sido observados de forma repetida en las estructuras de combustión cercanas a la pared del Abric en diferentes niveles arqueológicos (Carbonell et al., 2007; Vallverdú i Poch et al., 2010; Vallverdú i Poch et al., 2012). El tamaño de pocos decímetros concuerda con otras medidas realizadas en otros yacimientos, que también recogen el registro del inicio del uso generalizado del fuego por las poblaciones humanas arcaicas, como en el yacimiento de Kebara (Israel) (Meignen et al., 2001; Speth, 2006).

La gran estructura de combustión de la zona 010 muestra hogares elementales que interpretamos por

\footnotetext{
Figura 8. Documentación de campo de la estructura de combustión U-V / 50 - 53, de la que forma parte de la zona O10 (Chacón et al., 2015 ), dedicada a la planimetría de las litofacies antrópicas y la microestratigrafía del nivel O del Abric Romaní. La escala horizontal es la misma para todas las tres secciones estratigráficas y los valores de la profundidad de la escala vertical se refieren al punto cero del yacimiento. 1 , contorno de la extensión del depósito carbonoso. 2, contorno de la extensión de los sedimentos enrojecidos atribuidos a la rubefacción. a, arenas y gránulos de travertino estratificados en lechos enrojecidos horizontales. b, arenas y gránulos de travertino estratificados en lechos negros horizontales. c, arenas y gránulos de travertino estratificados en lechos horizontales de color gris y gris blanco. d, arenas y gránulos de travertino estratificados en lechos horizontales y masivos de color amarillo. e, arenas cementadas y travertinos estratificados de color amarillo o enrojecido en lechos horizontales o en forma de domo. f, fragmentos de travertino de la talla grava y bloque. Figure 8. Field record of combustion structure U-V / 50 - 53, which forms part of zone 010 (Chacón et al., 2015), used to draw up the Abric lithofacies map and determine the microstratigraphy of Abric Romani level $O$. The horizontal scale is the same for the three stratigraphic profiles and the deep values of the vertical scale are related to zero level of the archaeological site. 1, outline of the charcoal-rich deposits. 2 , outline of the reddened deposits. a, reddened travertine sands and granules in stratified horizontal beds. $b$, travertine sands and granules in black stratified horizontal beds. $c$, travertine sands and granules in grey and whitish (milky) grey stratified horizontal beds. $d$, travertine sands and granules stratified in yellow horizontal beds. e, cemented sands and travertines stratified in yellow or red dome-shaped or horizontal beds. $f$, gravel and blocks of travertine.
} 

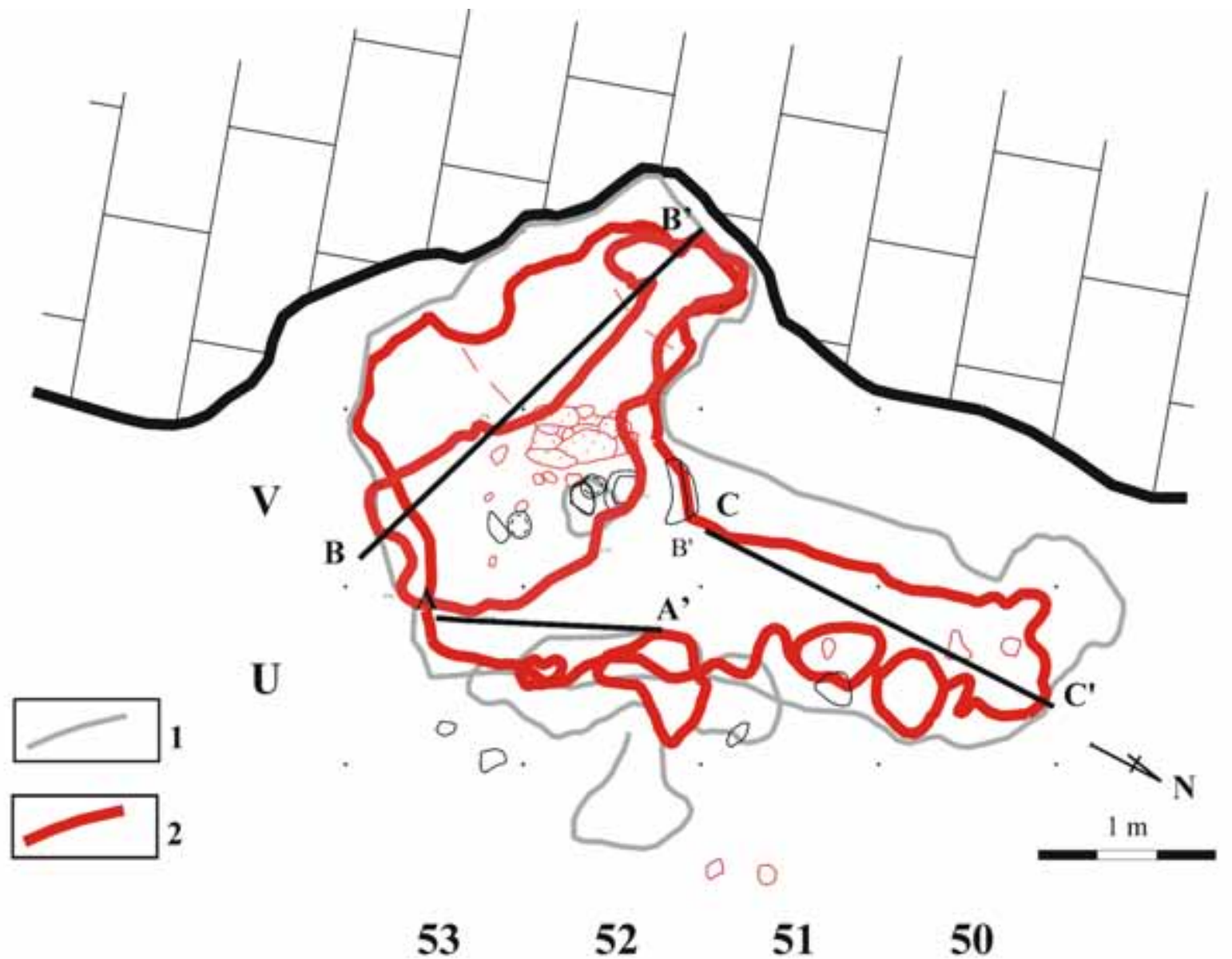

51

50
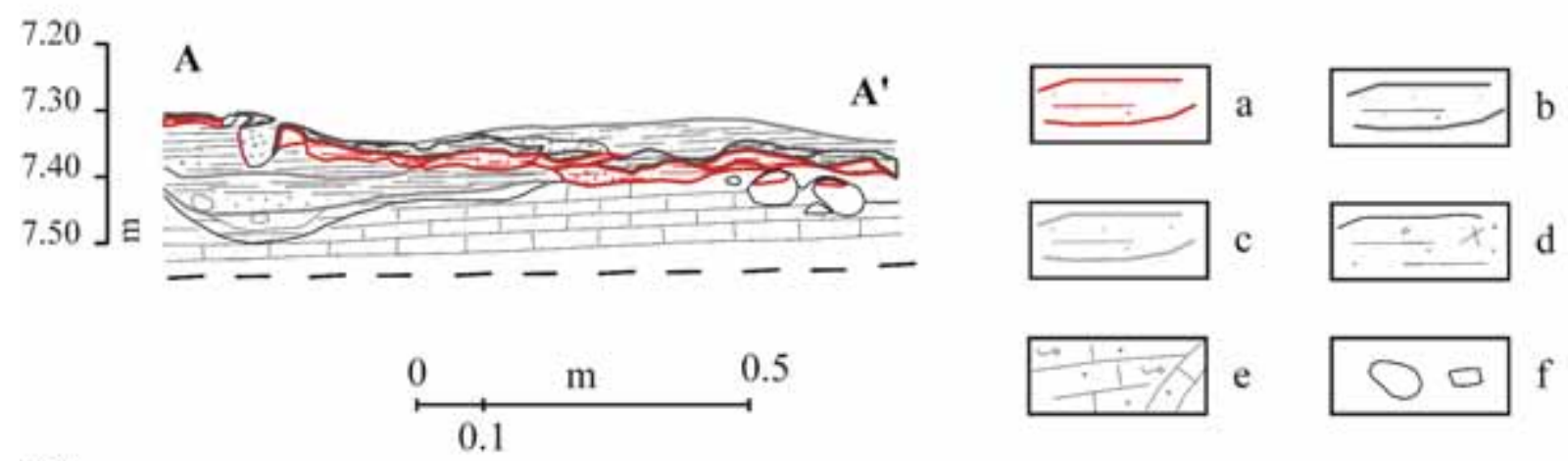

\section{$\left.\begin{array}{l}7.20 \\ 7.30 \\ 7.40 \\ 7.50 \\ 7.60\end{array}\right]$ ]}
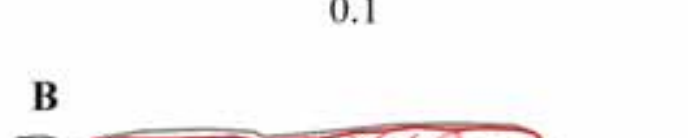
caracterizar el uso del fuego repetido en una zona de dormitorio y descanso. La visibilidad arqueológica de esta zona de dormitorio y descanso, por la imbricación y acreción lateral de hogares elementales pequeños, no es similar a la descubierta en el nivel $\mathrm{N}$ (Fig. 4). Aunque en alguna de las secciones microestratigráficas de la figura 8 (la sección $B-B^{\prime}$ ) aún conserva un espaciado entre grupos de hogares elementales similar a la distancia entre estructuras de combustión de las área dormitorio del nivel N. Entre los dos grupos de hogares elementales existen depósitos carbonosos sin suela de rubefacción que pueden ser asimilados a facies carbonosas heterogéneas relacionadas con los procesos de dispersión de los hogares elementales agrupados (depósitos secundarios).

Nuestra aproximación es comparativa y basada en la guía de la planimetría de los modelos etnológicos. El tiempo etnológico y arqueológico, y por decirlo de alguna manera también el tiempo litoestratigráfico, tiene sentido para fundamentar la analogía ya que proporciona el tiempo óptimo para ser visible en el registro arqueológico. Las observaciones etnoarqueológicas también consideran que los eventos pompeyanos, los instantes en el tiempo etnográfico, poseen una visibilidad arqueológica imperceptible (Brooks and Yellen, 1987).

El conjunto arqueológico del nivel $\mathrm{N}$ (como conjunto en el que se incluyen restos y estructuras arqueológicas) posee una visibilidad nítida para la analogía etnológica. La visibilidad arqueológica de la zona dormitorio y de descanso 010 del nivel O sólo permite hacer una analogía forzada por la gran cantidad de eventos de uso del fuego observados en las secciones microestratigraficas de la estructura de combustión. Pero las características constructivas y la distribución espacial de sus hogares elementales agrupados permiten indicar un uso del fuego determinado y distintivo entre el registro de las estructuras de combustión de los asentamientos prehistóricos del Abric (Sañudo et al., 2012; Vallverdú i Poch y Courty, 2012; Vallverdú i Poch et al., 2010; Vallverdú i Poch et al., 2012).

\section{Conclusiones}

En este manuscrito hemos tratado los resultados basados en la descripción multiescalar (de campo y microscópica) de sedimentos muestreados en estructuras de combustión. Los depósitos de ocupación producidos por el uso del fuego han intentado ser discutidos a la luz de los patrones de asentamiento fundamentados en la identificación de áreas de actividad prehistóricas en el registro arqueológico. $\mathrm{Ha}$ sido utilizado el estudio de caso basado en el registro espacial y microestatigrafico de las estructuras de combustión del nivel $\mathrm{N}$ ya publicado (Vallverdú i Poch et al., 2010). A este estudio hemos introducido las observaciones microscópicas de muestras en lámina delgada para argumentar diferentes usos del fuego en el nivel N. Esta investigación propone, en definitiva, que los yacimientos con depósitos de ocupación humanos pueden promover nuevos planteamientos en torno a cómo los arqueólogos desenterramos los depósitos arqueológicos y por qué exploramos los conjuntos arqueológicos.

Las investigaciones de los depósitos sedimentarios culturales abarcan, por citar los principales (Goldberg and Mcphail, 2006): depósitos de ocupación producidos y afectados por el uso del fuego; depósitos de ocupación de basureros (depósitos terciarios) de distintos componentes (vaciado de hogares, de limpieza de grasas, coprolitos, huesos, etc.); depósitos de ocupación y naturales afectados por la compactación y la dispersión producida por la circulación humana, bajo diferentes condiciones microambientales y clase de materiales sedimentarios (sobre superficies secas y húmedas, sobre superficies cubiertas por diferentes tejidos, sobre tierra batidas o pavimentos preparados mediante materiales de construcción, sobre depósitos de disgregación de paredes de casas - habitación y tipos de cubierta, etc.). En los estudios geoarqueológicos recientes también han surgido investigaciones interesadas en la modificación de los sedimentos de los paisajes agrícolas y urbanos (la fertilización, la irrigación, la labranza, las tierras negras urbanas, etc.). La representación espacial de las facies y microfacies de estos litotopos constituidos por depósitos de ocupación, mediante metodologías de muestreo en las superficies excavadas de los yacimientos, puede ser representada dentro de la cartografía del asentamiento prehistórico (Vallverdú i Poch and Courty, 2012). Una de las ventajas de trabajar en la Arqueología prehistórica de las poblaciones arcaicas, además de su redundancia espacial que mejora su visibilidad arqueológica para la analogía etnológica, es documentar su patrón de asentamiento y compararlo con los asentamientos de los cazadores-recolectores actuales. Desde un punto de vista evolutivo y conductual, estos asentamientos humanos en el que se generaliza el uso del fuego (el hogar) no son tan diferentes de las poblaciones modernas actuales. Las evidencias proporcionadas por el estudio de los sedimentos antrópicos son también sin duda una productiva línea de inspiración para la Arqueología experimental (March et al., 2014). 


\section{Agradecimientos}

En los trabajos de campo e investigación en el Abric Romaní participan un gran número de promociones de estudiantes del grado de Geografia e Historia y del master Cuaternario y Prehistoria de la Universidad Rovira i Virgili de Tarragona. También destacar el trabajo de investigadores afines a la Geología del Cuaternario en este proyecto de investigación y excavación, y entre ellos me gustaría señalar el apoyo y acogida recibido por el Dr. Ramón Julià, del Institut Jaume Almera de Barcelona cuando empecé a trabajar en el proyecto de excavación e investigación del Abric Romaní. A todos ellos, mi sincero agradecimiento. Finalmente, agradecer la invitación e interés de los editores de este BGM por el proyecto del Abrigo Romaní; y también mi reconocimiento a los editores y revisores por su tiempo dedicado a mejorar este manuscrito.

\section{Referencias}

Adderley, W. P., Wilson, C.A., Simpson, I.A. and Davidson, D.A. 2010. Anthropogenic Features. In: Stoops, G., Marcelino, V. and Mees, F. (eds.) Interpretation of micromorphological features of soils and regoliths. Elsevier, Amsterdam, 569-588.

Adouze, F. 1987. Des modèles et des faits : les modèles de A. Leroi-Gourham et de L. Bindford confrontés aux résultats récents. Bulletin de la Société Prehistorique Française, 84, 343-352.

Arteaga, I., Allué, E., Pastó, I., Vallverdú i Poch, J. and Carbonell, E. 2001a. Els fogars del paleolític mitjà de I'Abric Romaní (Capellades, Anoia). Cypsela, 13, 11-27.

Arteaga, I., Angelucci, D.E., Carbonell, E., Courty, M.A. and Vallverdú i Poch, J. 2001b. Microfacies and sedimentary environment of the Upper-Middle Palaeolithic Boundary at Abric Romani (Capellades, Barcelona). Cuaternario $y$ Geomorfología, 15, 15-30.

Bailey, G. 2007. Time perspectives, palimpsests and the archaeology of time. Journal of Anthropological Archaeology, 26, 198-223.

Balter, M. 2009. Better Homes and Hearths, NeandertalStyle. Science, 326, 1056-1057.

Bartroli, R., Cebrià, R., Muro, I., Riu, E. and Vaquero, M. 1995. A frec de ciència. L'Atles d'Amador Romani $i$ Guerra. Ajuntament de Capellades, Capellades, 232 pp.

Binford, L.R. 1987. Researching Ambiguity: Frames of Reference and Site Structure. In: Kent, S. (ed.) Method and Theory for Activity Area Research. Columbia University Press, New York, 449-512.

Binford, L.R. 1988. En busca del pasado. Descifrando el registro arqueológico. Crítica, Barcelona, $282 \mathrm{pp}$.

Binford, L.R. 1996. Hearth and home: the spatial analysis of ethnographically documented rock shelter occupations as a template for distinguishing between human and hominid use of sheltered space. Proceedings of the XIII
UISPP Congress. Middle Palaeolithic and Middle Stone Age Settlement Systems, Forli., 229-239.

Bischoff, J.L., Julià, R. and Mora, R. 1988. Uranium-Series Dating of the Mousterian Occupation at Abric Romani, Spain. Nature, 332, $68-70$.

Bischoff, J.L., Ludwing, K., Garcia, J.F., Carbonell, E., Vaquero, M. and Stafford, T.W. 1994. Dating of the basal Aurignacian Sandwich at Abric Romani (Catalunya, Spain) by Radiocarbon and Uranium-Series. Journal of Archaeological Science, 21, 541-551.

Blunier, T. and Brook, E.J. 2001. Timing of Millenial-Scale Climate Change in Antarctica and Greenland During the Last Glacial Period. Science, 291, 109-112.

Bordes, F. 1975. Sur la notion de sol d'habitat en préhistoire paléolithique. Bulletin de la Société Prehistorique Française, 72, 139-144.

Brochier, J.E. 1983a. Bergeries et feux de bois néolithiques dans le midi de la France. Caractérisation et incidence sur le raisonnement sédimentologique. Quatär, 33/34, 181-193.

Brochier, J.E. 1983b. Combustion et parcage des herbivores domestiques. Le point de vue du sédimentologue. Bulletin de la Société Préhistorique Française., 80, 143145.

Brochier, J.E. 1996. Feuilles ou fumiers? Observations sur le rôle des poussières sphérolitiques dans la interpretation des dépôts archéologiques holocènes. Anthropozoologica, 24.

Brochier, J.E. and Thinon, M. 2003. Calcite crystals, starch grains aggregates or ... POCC? Comment on 'calcite crystals inside archaeological plant tissues'. Journal of Archaeological Science, 30, 1211-1214.

Brooks, A.S. and Yellen, J.E. 1987. The Preservation of Activity Areas in the Archaeological Record: Ethnoarchaeological and Archaeological Work in Northwest Ngamiland, Botswana. In: Kent, S. (ed.) Method and Theory for Activity Area Research. An Ethnoarchaeological Approach. Columbia University Press, New York, 63-106.

Bullock, P., Fedoroff, N., Jonguerius, A., Stoops, G. and Tursina, T. 1985. Handbook for Soil Thin Section Description. Waine Research Publications, Wolverhampton, $152 \mathrm{pp}$.

Burjachs, F. and Julià, R. 1994. Abrupt Climatic Changes during the Last Glaciation Based on Pollen Analysis of the Abric Romani, Catalonia, Spain. Quaternary Research, 42, 308 - 315.

Butzer, K. W. 1989. Arqueología. Una ecología del hombre. Bellaterra, Barcelona, 345 pp.

Campbell, C.V. 1967. Lamina, laminaset, bed and bedset. Sedimentology, 8, 7-26.

Canti, M. and Linford, N. 2000. The effects of Fire on Archaeological Soils and Sediments: Temperature and Colour Relationships. Proceedings of the Prehistoric Society, 66, 385-395.

Canti, M.G. 2003. Aspects of the chemical and microscopic characteristics of plant ashes found in archaeological soils. Achievements in Micromorphology, 54, 339-361.

Carbonel, E., Giralt, S. and Vaquero, M. 1994. Abric Romani (Capellades, Barcelone, Espagne) : une importante 
Vallverdú i Poch, J., 2018. Investigación geoarqueológica en los depósitos de ocupación... Boletín Geológico y Minero, 129 (1/2): $129-152$

séquence anthropisée au Pléistocène supérieur. Bulletin de la Société Prehistorique Française, 91, 47-55.

Carbonell, E. 1992. Abric Romaní, nivell H: un model d'estratègia ocupacional al Plistocè superior mediterrani. Estrat, 5, 159-308.

Carbonell, E. 2002. Abric Romaní nivell I. Models d'ocupació de curta durar de fa 46.000 anys a la Cinglera del Capelló (Capellades, Anoia, Barcelona). Universitat Rovira i Virgili, Tarragona, 323 pp.

Carbonell, E., Cebrià, A., Allué, E., Caceres, I., Castro, Z., Diaz, R., Esteban, M., Ollé, A., Pasto, I., Rodriguez, X. P., Rosell, J., Sala, R., Vallverdu i Poch, J., Vaquero, M. and Vergés, J. M. 1996. Behavioural and Organizational Complexity in the Middle Palaeolithic From The Abric Romani. In: Carbonell, E. and Vaquero, M. (eds.), The Last Neandertals. The First Anatomically Modern Humans. Universitat Rovira i Virgili, Tarragona, 385-434.

Carbonell, E., Cebrià, A., Esteban, A., Móra, R. and Parra, I. 1983. Aproximació crítica a l'estudi dels espais prehistòrics. Estudis d'Història Agrària, 3, 87-99.

Carbonell, E., Lorenzo, C. and Vallverdu i Poch, J. 2007. Centralidad espacial y operativa de los neanderthales. Análisis espacial diacrónico de las actividades de combustión en el Abric Romaní (Capellades, Anoia, Barcelona). In: Baquedano, E. (ed.), Universo Neanderthal 1. Fundación Duques de Soria, Soria, 197219.

Carbonell i Roura, E. 2012. High resolution archaeology and Neanderthal behavior: time and space in level J of Abric Romaní (Capellades, Spain). Springer, Dordrecht, 411 pp.

Chacón, M.G., Bargalló, A., Gabucio, M. J. and Vaquero, M. 2015. Neanderthal Behaviors from a Spatio-Temporal Perspective: An Interdisciplinary Approach to Interpret Archaeological Assemblages. In: Conard, N.J. and Delagnes, A. (eds.), Settlement Dynamics of the Middle Paleolithic and Middle Stone Age, Volume IV. Kerns Verlag, Tübingen, 253-294.

Chacón, M.G., Vaquero, M. and Carbonell, E. 2012. The Neanderthal Home: Spatial and Social Behaviours. The Neanderthal Home: spatial and social behaviours, 247, 19.

Coudart, A. 2002. Sobre las analogía etnográfica y la Etnoaqueología y acerca de la Historia de las relaciones entre Arqueología y Etnología. In: Garanger, J., Chavaillon, J. and Leroi-Gourhan, A. (eds.), La Prehistoria en el mundo. Akal Ediciones, Tres Cantos, Madrid, 221-234.

Courty, M.A. 1984. Interprétation des aires de combustion par la micromorphologie. Bulletin de la Société Prehistorique Française, 80, 169-171.

Courty, M.A., Goldberg, P. and Macphail, R.I. 1989. Soils and Micromorphology in Archaeology. Cambridge University Press, Cambridge, 344 pp.

Courty, M.A., Goldberg, P. and Macphail, R.I. 1994. Ancient People - Lifestyles and Cultural Patterns. In: Wilding, L. and Oleshko, K. (eds.), Micromorphological Indicators of Anthropogenic Effets on Soils. ISSS, 15th International Conference of Soil Science, Acapulco, Mexico, 250-269.

David, F., Julien, M. and Karlin, C. 1973. Approche d'un niveau archéologique en sédiment homogène. In: Collectif (ed.), L'homme, hier et aujourd'hui : recueil d'études en hommage à André Leroi-Gourhan. Cujàs, Paris, 65-72.

Dibble, H.L., Chase, P.G., McPherron, S.P. and Tuffreau, A. 1997. Testing the Reality of a "Living Floor" with Archaeological Data. American Antiquity, 62, 629-651.

Fisher, J.W. and Strickland, H.C. 1991. Dwellings and fire places: keys to Efe Pygmy campsite structure. In: Gamble, C. and Boismier, W.A. (eds.), Ethnoarchaeologial Approaches to Mobile Campsites. International Monographs in Prehistory, Ann Arbor, 215236.

Folch, R. 1981. La vegetació dels països catalans. Ketres, Barcelona, 513 pp.

Galanidou, N. 2000. Patterns in Caves: Foragers, Horticulturists, and the Use of Space. Journal of Anthropological Archaeology, 19, 243 - 275.

Gé, T., Courty, M.A., Mattews, W. and Wattez, J. 1993. Sedimentary Formation Processes of Occupation Surfaces. In: Goldberg, P., Nash, D. and Petraglia, M.D. (eds.), Formation Processes in Archaeological Context. Prehistory Press, Madison, $149-163$.

Giralt, S. and Julià, R. 1996. The Sedimentary Record of the Middle-Upper Palaeolithic transition in the Capellades Area (NE Spain). In: Carbonell, E. and Vaquero, M. (eds.), The Last Neandhertals. The First Anatomically Modern Humans. Universitat Rovira i Virgili, Tarragona, 365 -376.

Goldberg, P., Lev-Yadun, S. and Bar-Yosef, O. 1994. Petrographic Thin Sections of Archaeological Sediments: A New Method for Paleobotanical Studies. Geoarcheology, 9, 243-257.

Goldberg, P. and Macphail, R.I. 2010. Archaeological Materials. In: Stoops, G., Marcelino, V. and Mees, F. (eds.), Interpretation of micromorphological features of soils and regoliths. Elsevier, Amsterdam, 589-622.

Goldberg, P. and Mcphail, R.I. 2006. Practical andTheoretical Geoarchaeology. Blackwell Science, Malden, 454 pp.

Goldberg, P., Nash, D. and Petraglia, M.D. 1993. Formation Processes in Archaeological Context. Prehistory Press, Madison, Massachusetts, $188 \mathrm{pp}$.

Goldberg, P. and Whitbread, I. 1993. Micromorphological studies of Bedouin tent floors. In: Goldberg, P., Nash, D.T. and Petraglia, M.D. (eds.), Formation Process in Archaeological Context. Prehistory Press, Madison, Massachusetts, 165-188.

Goren-Inbar, N., Alperson, N., Kislev, M., Simchoni, O., Melamed, Y. Ben-Nun, A. and Werker, E. 2004. Evidence of Hominin Control of Fire at Gesher Benot Ya`aqov, Israel. Science, 304, 725-727.

Gowlett, J.A.J. 1997. High definition archaeology: Ideas and evaluation. World Archaeology, 29, 152-171.

Henry, D. 2012. The palimpsest problem, hearth pattern analysis, and Middle Paleolithic site structure. The Neanderthal Home: spatial and social behaviours, 247, 246-266.

Julià, R. 1983. Travertines. In: Scholle, P.A., Bebout, D.G. and Moore, C.H. (eds.), Carbonate depositional environments. American Association of Petroleum Geologists, Tulsa, Okla., U.S.A., 64-72. 
Julien, M. 2002. Hacia la interpretación de los suelos de habitación. In: Garanger, J., Chavaillon, J. and LeroiGourhan, A. (eds.), La Prehistoria en el mundo. Akal Ediciones, Tres Cantos, Madrid, 195-220.

Kent, S. 1991. The Relationship between Mobility Strategies and Site Structure. In: Kroll, E. M. and Price, T. D. (eds.), The interpretation of Archaeological Spatial Patterning. Plenum Press, New York, 33-59.

March, R., Lucquin, A., Joly, D., Ferreri, J. and Muhieddine, M. 2014. Processes of Formation and Alteration of Archaeological Fire Structures: Complexity Viewed in the Light of Experimental Approaches. Journal of Archaeological Method and Theory, 21, 1-45.

Mattews, W. 1994. The imprint of living in a Mesopotamian city: questions and answers. In: Luff, R. and Rowley Conwy, P. (eds.), Whither Environmental Archaeology? Oxbow Books, Oxford, 171-212.

Meignen, L., Bar-Yosef, O., Goldberg, P. and Weiner, S. 2001. Le feu au Paléolithique moyen : recherches sur les structures de combustion et le statut des foyers. L'exemple du Proche-Orient. Paléorient, 26, 9-22.

Mentzer, S. 2014. Microarchaeological Approaches to the Identification and Interpretation of Combustion Features in Prehistoric Archaeological Sites. Journal of Archaeological Method and Theory, 21, 616-668.

Miedema, R. 1997. Applications of Micromorphology of Revelance to Agronomy. Advances in Agronomy, 59, 119-169.

Mora, R., Carbonell, E., Cebrià, A. and Martínez, J. 1988. Els sòls d'ocupació a l'abric Romaní (Capellades, Anoia). Tribuna d'Arqueologia, 1987 - 1988, 115 - 123.

Movius, H.L. 1966. The hearths of the upper Perigordian and Aurignacian horizons at the Abri Pataud, Les Eyzies (Dordogne), and their possible significance. American Anthropologist, 68, 296-325.

Muro, I., Mora, R., Carbonell, E. and Cebria, A. 1987. Ensayo de interpretación del marco geomorfológico de un yacimiento del Paleolítico Medio Catalán: Abric Romaní (Capellades, Anoia). Cypsela, VI, 125 - 131.

Renfrew, C. 1976. Archaeology and the earth sciences. In: Davidson, D. A. and Shackley, M. C. (eds.), Geoarchaeology. Earth science and the past. Duckworth, London, 1-7.

Rigaud, J.P., Simek, J. and Ge,T. 1995. Mousterian fires from Grotte XVI. Antiquity., 69, 902-912.

Roebroeks, W. and Villa, P. 2011. On the earliest evidence for habitual use of fire in Europe. Proceedings of the National Academy of Sciences, 108, 5209-5214.

Sala, R., Ed. (2014). Los cazadores recolectores del Pleistoceno y del Holoceno en Iberia y el estrecho de Gibraltar: estado actual del conocimiento del registro arqueológico. Servicio de Publicaciones Universidad de Burgos y Fundación Atapuerca, Burgos, 765 pp.

Salomon, J.N. 1997. Les influences climatiques sur la géomorphologie karstique: exemple des milieux tropicaux et arides. Quaternaire, 8, 107-117.

Sandgathe, D.M., Dibble, H.L., Goldberg, P., McPherron, S.P., Turq, A., Niven, L. and Hodgkins, J. 2011. Timing of the appearance of habitual fire use. Proceedings of the National Academy of Sciences, 108, E298-E298.
Sañudo, P., Vallverdú i Poch, J. and Canals, A. 2012. Spatial Patterns in Level J. In: Carbonell i Roura, E. (ed.), High Resolution Archaeology and Neanderthal Behavior. Springer Netherlands, 47-76.

Schiffer, M.B. 1987a. Cultural deposition. Formation Processes of the Archaeological Record. University of Utah Press, Salt Lake City, 47-98.

Schiffer, M.B. 1987b. Formation Processes of the Archaeological Record. University of the New Mexico Press, Alburquerque, 428 pp.

Schiffer, M.B. 1995. Behavioral Archaeology. First Principles. University of Utah Press, Salt Lake City, 289 pp.

Shahack-Gross, R. 2008. Approaches to understanding formation of archaeological sites in Israel: Materials and processes. Israel Journal of Earth Sciences, 56, 73-86.

Solé Sabarís, L. 1956. Entre la Geología y la Historia. Universidad de Barcelona, Barcelona, 70 pp.

Speth, J. 2006. Housekeeping, Neandertal-Style Hearth Placement and Midden Formation in Kebara Cave (Israel). In: Hovers, E. and Kuhn, S. (eds.), Transitions Before the Transition. Springer US, 171-188.

Stein, J.K. 2001. A Review of site Formation Processes and Their Relevance to Geoarchaeology. In: Goldberg, P., Holliday, V.T. and Ferring, C.R. (eds.), Earth Sciences and Archaeology. Kluwer Academic / Plenum Publishers, New York, 37-48.

Stein, J.K. and Farrand, W.R. 1985. Archaeological Sediments in Context. Center for the Study of Early Man, University of Maine, Maine, $147 \mathrm{pp}$.

Stein, J.K. and Rapp, G. 1985. Archaeological Sediments: a largely Untapped Reservoir of Information. In: Wilkie, N. C. and Coulson, W. D. E. (eds.), Contributions to Aegean Archaeology. Center for Ancient Studies. University of Minnesota, Minneapolis, 143-159.

Stoops, G. 2003. Guidelines for analysis and description of soil and regolith thin sections. Soil Science Society of America, Madison, 184 pp.

Vallverdú i Poch, J. and Courty, M.A. 2012. Microstratigraphic Analysis of Level J Deposits: A Dual Paleoenvironmental-Paleoethnographic Contribution to Paleolithic Archeology at the Abric Romaní. In: Carbonell i Roura, E. (ed.), High Resolution Archaeology and Neanderthal Behavior. Springer Netherlands, 77-133.

Vallverdú i Poch, J., Gómez de Soler, B., Vaquero, M. and Bischoff, J. 2012. The Abric Romaní Site and the Capellades Region. In: Carbonell i Roura, E. (ed.), High Resolution Archaeology and Neanderthal Behavior. Springer Netherlands, 19-46.

Vallverdú i Poch, J., Allué, E., Bischoff, J. L., Cáceres, I., Carbonell, E., Cebrià, A., García-Antón, D., Huguet, R., Ibáñez, N., Martínez, K., Pastó, I., Rosell, J., Saladié, P. and Vaquero, M. 2005a. Short human occupations in the Middle Palaeolithic level i of the Abric Romaní rock-shelter (Capellades, Barcelona, Spain). Journal of Human Evolution, 48, 157-174.

Vallverdú i Poch, J., Alonso, S., Bargalló, A., Bartrolí, R., Campeny, G., Carrancho, Á., Expósito, I., Fontanals, M., Gabucio, J., Gómez, B., Prats, J. M., Sañudo, P., Solé, À., Vilalta, J. and Carbonell, E. 2012. Combustion structures of archaeological level $\mathrm{O}$ and mousterian activity areas 
with use of fire at the Abric Romaní rockshelter (NE Iberian Peninsula). Quaternary International, 247, 313-324. Vallverdú i Poch, J., Vaquero, M., Cáceres, I., Allué, E., Rosell, J., Saladié, P., Chacón, G., Ollé., A., Canals, A., Sala, R., Courty, M. A. and Carbonell, E. 2010. Sleeping Activity Area within the Site Structure of Archaic Human Groups. Evidence from Abric Romaní Level N Combustion Activity Areas. Current Anthropology, 51, 137-145.

Vallverdú i Poch, J., Vaquero, M., Courty, M.A. and Carbonell, E. 2005b. Procesos sedimentarios rápidos y ocupaciones humanas poco frecuentes. La sección del Pleistoceno Superior de la Coveta Nord del Abric Romaní (Capellades, Anoia, Barcelona). In: Santonja, M., Pérez-González, A. and Machado, M. J. (eds.), Geoarqueología y Conservación del Patrimonio. ADEMA, Madrid, 20-26.

Vaquero, M. 2008. The history of stones: behavioural inferences and temporal resolution of an archaeological assemblage from the Middle Palaeolithic. Journal of Archaeological Science, 35, 3178-3185.

Vaquero, M. 2013. Análisis micro-espacial: áreas domésticas, variabilidad funcional y patrones temporales. In: García Díez, M. and Zapata, L. (eds.), Métodos y técnicas de análisis y estudio en arqueología prehistórica: de lo técnico a la reconstrucción de los grupos humanos. Servicio Editorial de la Universidad del País Vasco, Bilbao, 245-272.

Vaquero, M., Allué, E., Bischoff, J. L., Burjachs, F. and Vallverdú i Poch, J. 2013. Environmental, depositional and cultural changes in the Upper Pleistocene and early Holocene: the Cinglera del Capelló sequence (Capellades, Spain). Quaternaire, 24, 49-64.
Vaquero, M., Allué, E. and Vallverdú i Poch, J. 2012. Conclusions: Landscapes, Campsites, Time, and Neanderthal Behavior. In: Carbonell i Roura, E. (ed.), High Resolution Archaeology and Neanderthal Behavior. Springer Netherlands, 389-405.

Vaquero, M., Bargalló, A., Chacón, M. G., Romagnoli, F. and Sañudo, P. 2015. Lithic recycling in a Middle Paleolithic expedient context: Evidence from the Abric Romaní (Capellades, Spain). The Origins of Recycling: $A$ Paleolithic Perspective, 361, 212-228.

Vaquero, M. and Pasto, I. 2001. The Definition of Spatial Units in Middle Palaeolithic Sites: The Hearth-Related Assemblages. Journal of Archaeological Science, 28, 1209-1220.

Vidal, L. M. 1913. Abrich Romaní, Estació Agut, Cova de l’Or o dels Encantats. Estacions Prehistóriques de les époques musteriana, magdaleniana i neolítica a Capellades i Sta. Creu d'Olorde. Butlletí de la Institució Catalana d'Història Natural, IV, 267-302.

Wattez, J. 1988. Contribution à la conaissance des foyers préhistoriques par l'étude des cendres. Bulletin de la Société Prehistorique Française, 85, 352-366.

Wattez, J. 1990 Dynamique de Formation des Structures de Combustion de la Fin du Paléolithique au Néolitique Moyen. Approche Méthodologique et Implications Culturelles. Université de Paris I, Paris, 438. pp.

Weiner, S., Qinqi, X., Goldberg, P., Liiu, J. and Bar-Yosef, O. 1988. Evidence for the use of fire at Zhoukoudian, China. Science, 281, 251-253.

Weiner, S. 2010. Microarchaeology: beyond the visible archaeological record. Cambridge University Press, New York, 396 pp.

Recibido: abril 2015

Revisado: enero 2016

Aceptado: febrero 2016

Publicado: marzo 2018 